\title{
Automotive engine FDI by application of an automated model-based and data-driven design methodology
}

\author{
Carl Svärd, Mattias Nyberg, Erik Frisk and Mattias Krysander
}

\section{Linköping University Post Print}

\section{Tweet}

N.B.: When citing this work, cite the original article.

Original Publication:

Carl Svärd, Mattias Nyberg, Erik Frisk and Mattias Krysander, Automotive engine FDI by application of an automated model-based and data-driven design methodology, 2013, Control Engineering Practice, (21), 4, 455-472.

http://dx.doi.org/10.1016/j.conengprac.2012.12.006

Copyright: Elsevier

http://www.elsevier.com/

Postprint available at: Linköping University Electronic Press

http://urn.kb.se/resolve?urn=urn:nbn:se:liu:diva-77189 


\title{
Automotive Engine FDI by Application of an Automated Model-Based and Data-Driven Design Methodology
}

\author{
Carl Svärd ${ }^{\mathrm{a}, \mathrm{b}}$, Mattias Nyberg $^{\mathrm{b}}$, Erik Frisk ${ }^{\mathrm{a}}$, Mattias Krysander $^{\mathrm{a}}$ \\ ${ }^{a}$ Department of Electrical Engineering, Linköping University, SE-58183 Linköping, Sweden \\ ${ }^{b}$ Scania, Södertälje, Sweden
}

\begin{abstract}
Fault detection and isolation (FDI) in automotive diesel engines is important in order to achieve and guarantee low exhaust emissions, high vehicle uptime, and efficient repair and maintenance. This paper illustrates how a set of general methods for model-based sequential residual generation and data-driven statistical residual evaluation can be combined into an automated design methodology. The automated design methodology is then utilized to create a complete FDI-system for an automotive diesel engine. The performance of the obtained FDI-system is evaluated using measurements from road drives and engine testbed experiments. The overall performance of the FDI-system is good in relation to the required design effort. In particular no specific tuning of the FDI-system, or any adaption of the design methodology, were needed. It is illustrated how estimations of the statistical powers of the fault detection tests in the FDI-system can be used to further increase the performance, specifically in terms of fault isolability.
\end{abstract}

Keywords: fault diagnosis, fault detection, fault detection and isolation, FDI, automotive diesel engine

Email addresses: carl.svard@scania.com (Carl Svärd), mattias.nyberg@scania.com (Mattias Nyberg), frisk@isy.liu.se (Erik Frisk), matkr@isy.liu.se (Mattias Krysander) 


\section{Introduction}

Emission related legislations (United Nations, 2008; European Parliament, 2009; California EPA, 2010; United States EPA, 2009) require on-board diagnosis (OBD) of all faults in automotive engines that may lead to increased exhaust emissions. In addition, fault accommodation by, e.g., fault-tolerant control (FTC) (Blanke et al., 2006), and off-board diagnosis, are means in order to meet dependability requirements in the form of high vehicle uptime, high safety, and efficient repair. A necessity for both diagnosis and fault accommodation is fault detection and isolation (FDI).

Automotive engines pose several challenges and difficulties when it comes to the design of FDI-systems. Typically, engines are optimized for low-cost and high functionality, and not for FDI, which means that there is no hardware redundancy in the form of multiple sensors. To obtain good detection and isolation of faults it is therefore necessary to employ analytical redundancy and model-based FDI as long as the embedded control system has enough processing power. Moreover, the inherent complexity of automotive engines, as well as their multi-domain features due to chemical, mechanical, and thermodynamic subsystems, results in large-scale, dynamic, and highly non-linear models (Wahlström and Eriksson, 2011). Thus, such models must be handled by the methods used in the design of the FDI-system.

As a consequence of the complexity of automotive engines, in combination with their wide operating range, models are typically not fully capable of capturing their behaviour in all operating modes. This results in model errors, and in particular stationary model errors (Höckerdal et al., 2011a,b), regardless of substantial modelling work. In addition, a model may be more accurate in one operating mode than another and since the operating mode of the engine varies in time, so does the magnitude and nature of the model errors. These aspects must be taken into account in the design of the FDI-system.

It is clear that the design of a complete model-based FDI-system for an automotive engine, and for large-scale real-world systems in general, is an intricate 
task that demands a substantial engineering effort. An optimal solution in general requires detailed knowledge of the behaviour of the system and well-defined requirements, which are not available during early design stages. In order to make the overall design process more systematic and efficient, and in this way enable re-design or re-configuration, and eventually higher quality, a generic automated methodology for the design of FDI-systems has been developed.

The design methodology relies on previously developed methods for sequential residual generation (Svärd and Nyberg, 2010; Svärd et al., 2011a; Svärd, 2012) and statistical residual evaluation (Svärd et al., 2011b; Svärd, 2012). The residual generation methods described in Svärd and Nyberg (2010); Svärd et al. (2011a); Svärd (2012) are together able to design residual generators for fault detection and isolation in systems described by complex large-scale models. This was demonstrated in Svärd and Nyberg (2012), where they were combined with a residual evaluation approach based on the Kullback-Leibler divergence (Kullback and Leibler, 1951) and applied to the Wind Turbine Benchmark (Fogh Odgaard et al., 2009). The residual evaluation approach employed in Svärd and Nyberg (2012) was however not able to fully handle the issue concerning time-varying uncertainties related to model errors and operating modes discussed above. In this work, the automated design methodology is refined by means of the data-driven statistical residual evaluation approach described in Svärd (2012), which is able to handle this issue.

This paper illustrates how an FDI-system for an automotive diesel engine can be designed by application of this automated design methodology. The overall aim, and the main contribution, is to demonstrate how a set of general methods may be combined into a complete methodology in order to solve a real industrial problem, in this case, the challenging problem of automotive engine FDI (Nyberg and Stutte, 2004). In this sense, this work serves as an illustration of the state-of-practice in model-based FDI, and in particular sequential residual generation, e.g., Staroswiecki and Declerck (1989); Cassar and Staroswiecki (1997); Staroswiecki (2002); Pulido and Alonso-González (2004); Ploix et al. (2005); Travé-Massuyès et al. (2006); Blanke et al. (2006); Svärd 
and Nyberg (2010), and statistical residual evaluation, e.g., Willsky and Jones (1976); Gertler (1998); Basseville and Nikiforov (1993); Peng et al. (1997); AlSalami et al. (2006); Blas and Blanke (2011); Wei et al. (2011); Svärd et al. (2011b); Caponetti et al. (2011). Moreover, as a secondary contribution, the usefulness and properties of the specific methods described in Svärd and Nyberg (2010); Svärd et al. (2011a,b); Svärd (2012) are illustrated and discussed. For instance, it is empirically shown how the usage of residual generators utilizing both integral and derivative causality, i.e., mixed causality, increases the fault isolability, and how time-varying model errors can be handled in the framework of statistical likelihood-based residual evaluation.

The paper is structured as follows. Section 2 presents the automotive diesel engine system considered and the model of the system used in the design of the FDI-system. Section 3 gives an overview of the different stages in the automated design methodology from the user perspective. The different methods and their key properties are briefly discussed but technical details are kept at a minimum. Full details can be found in Svärd and Nyberg (2010); Svärd et al. (2011a,b); Svärd (2012). Sections 4 and 5 describe how the automated methodology was applied to the diesel engine system and discuss details and different aspects of the resulting FDI-system. In Section 6, the FDI-system is experimentally evaluated and some final remarks are given in Section 7.

\section{Automotive Diesel Engine System}

The system considered in this work is a 13-liter six-cylinder Scania truck diesel engine equipped with Exhaust Gas Recirculation (EGR), Variable Geometry Turbochargers (VGT), and intake throttle. A schematic of the system is shown in Figure 1. This section describes the system and the model used in the design of the proposed FDI-system.

\subsection{System Description}

Consider Figure 1. Air of temperature $T_{\mathrm{bc}}$ and pressure $p_{\mathrm{bc}}$ enters the system and passes the compressor side of the VGT. The compressed air, with mass-flow 


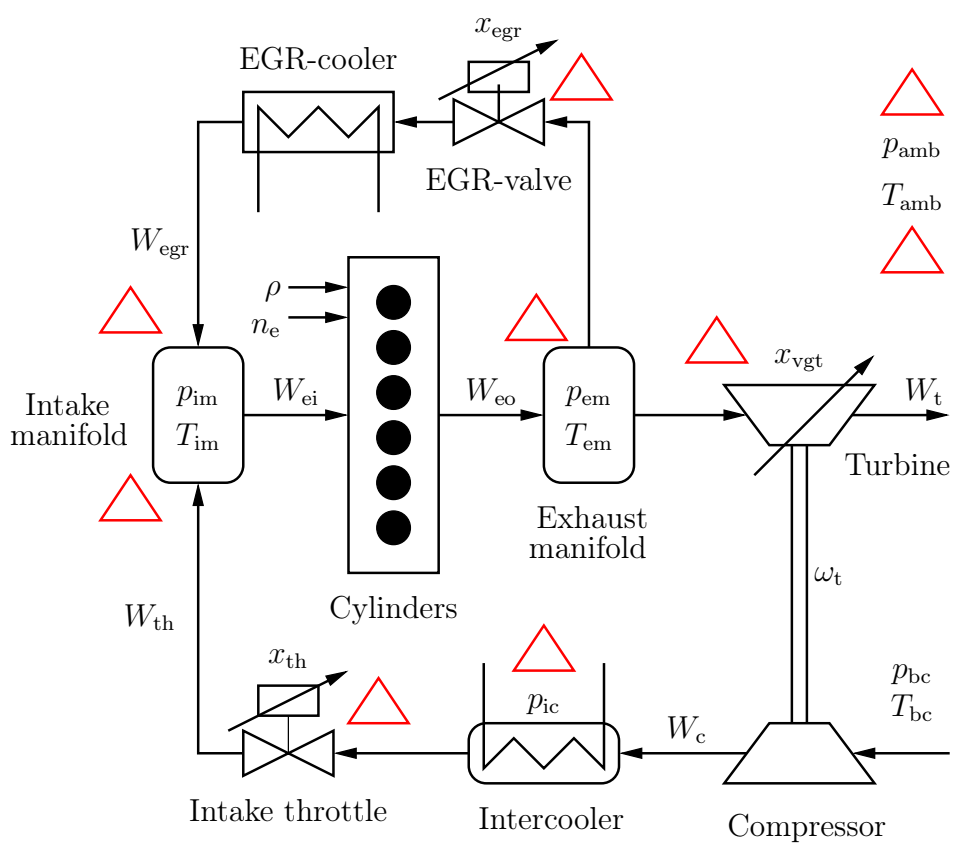

Figure 1: Schematic of the automotive diesel engine system. Locations of considered faults are illustrated with triangles.

$W_{\mathrm{c}}$, then enters the intercooler. The pressure of the air after the intercooler is denoted $p_{\text {ic }}$. The cooled air then passes the intake throttle, whose position is given by $x_{\mathrm{th}}$, and which is used to control the amount of air entering the intake manifold.

The air mass-flow after the intake throttle is denoted $W_{\text {th }}$, and the pressure and temperature of the air in the intake manifold are denoted $p_{\mathrm{im}}$ and $T_{\mathrm{im}}$, respectively. In the intake manifold, the air is mixed with recirculated exhaust gases, whose mass-flow is denoted $W_{\text {egr }}$, before it enters the cylinders. The amount of recirculated gas is controlled by the EGR-valve, whose position is denoted $x_{\text {egr }}$. The total mass-flow of the gas entering the cylinders is denoted $W_{\mathrm{ei}}$.

In the cylinders, the gas is mixed with fuel and then combusted. The amount of fuel injected into the cylinders is given by $\rho$, and the rotational speed of the engine is denoted $n_{\mathrm{e}}$. After the combustion, the gas enters the exhaust 
Table 1: Sensors and actuators in the diesel engine system.

\begin{tabular}{ll}
\hline Signal & Description \\
\hline$u_{x_{\mathrm{th}}}$ & Throttle position actuator \\
$u_{x_{\mathrm{egr}}}$ & EGR-valve position actuator \\
$u_{x_{\mathrm{vgt}}}$ & VGT-valve position actuator \\
$u_{\rho}$ & Injected fuel actuator \\
$y_{n_{\mathrm{e}}}$ & Engine speed sensor \\
$y_{p_{\mathrm{amb}}}$ & Ambient temperature sensor \\
$y_{T_{\mathrm{amb}}}$ & Ambient pressure sensor \\
$y_{p_{\mathrm{ic}}}$ & Inter-cooler pressure sensor \\
$y_{p_{\mathrm{im}}}$ & Inlet manifold pressure sensor \\
$y_{T_{\mathrm{im}}}$ & Inlet manifold temperature sensor \\
$y_{p_{\mathrm{em}}}$ & Exhaust manifold pressure sensor \\
\hline
\end{tabular}

manifold. The mass-flow of the exhaust gas is denoted $W_{\text {eo }}$, and the pressure and temperature of the gas in the exhaust manifold $p_{\mathrm{em}}$ and $T_{\mathrm{em}}$, respectively. The exhaust gas then passes the turbine side of the VGT, whose rotational speed is given by $\omega_{\mathrm{t}}$, and leaves the system with mass-flow $W_{\mathrm{t}}$. The geometry of the VGT is controlled with the VGT-valve, whose position is denoted $x_{\mathrm{vgt}}$.

\subsection{Sensors and Actuators}

The system is equipped with 4 actuators, $u_{x_{\mathrm{th}}}, u_{x_{\mathrm{egr}}}, u_{x_{\mathrm{vgt}}}, u_{\rho}$, and 7 sensors, $y_{p_{\mathrm{amb}}}, y_{T_{\mathrm{amb}}}, y_{p_{\mathrm{ic}}}, y_{p_{\mathrm{im}}}, y_{T_{\mathrm{im}}}, y_{p_{\mathrm{em}}}, y_{n_{\mathrm{e}}}$. See Table 1 for details.

\subsection{Faults}

Faults in all sensors and actuators in Table 1, except in actuator $u_{\rho}$ and sensor $y_{n_{\mathrm{e}}}$, are considered. All faults along with their description can be found in Table 2. The approximate location of the faults are marked with triangles in Figure 1.

\subsubsection{Modeling of Faults}

The faults are modeled as additive signals in the corresponding equations in the nominal model presented in next section. For example, fault $\Delta_{y_{p_{\mathrm{im}}}}$, representing a fault in the intake manifold pressure sensor $y_{p_{\text {im }}}$, is modeled by simply adding $\Delta_{y_{p_{\mathrm{im}}}}$ to the equation describing the relation between the sensor value $y_{p_{\mathrm{im}}}$ and the actual intake manifold pressure $p_{\mathrm{im}}$, i.e., $y_{p_{\mathrm{im}}}=p_{\mathrm{im}}+\Delta_{y_{p_{\mathrm{im}}}}$. 
Table 2: List of considered faults. With some abuse of notation, a variable will denote both the fault mode and also later the variable used to model the fault behavior. Thus, there is a fault in the ambient pressure sensor, i.e., we are in fault mode $\Delta_{y_{p_{\mathrm{amb}}}}$ when the corresponding model variable is non-zero.

\begin{tabular}{ll}
\hline Fault & Description \\
\hline$\Delta_{y_{p_{\mathrm{amb}}}}$ & Fault in ambient pressure sensor \\
$\Delta_{y_{T_{\mathrm{amb}}}}$ & Fault in ambient temperature sensor \\
$\Delta_{y_{p_{\mathrm{ic}}}}$ & Fault in intercooler pressure sensor \\
$\Delta_{y_{p_{\mathrm{im}}}}$ & Fault in intake manifold pressure sensor \\
$\Delta_{y_{T_{\mathrm{im}}}}$ & Fault in intake manifold temperature sensor \\
$\Delta_{y_{p_{\mathrm{em}}}}$ & Fault in exhaust manifold pressure sensor \\
$\Delta_{u_{x_{\mathrm{th}}}}$ & Fault in throttle position actuator \\
$\Delta_{u_{x_{\mathrm{egr}}}}$ & Fault in EGR-valve position actuator \\
$\Delta_{u_{x_{\mathrm{vgt}}}}$ & Fault in VGT-valve position actuator \\
\hline
\end{tabular}

The main argument for using this fault modeling approach is that it is considered to be hard, or even impossible, to know how a faulty component behaves in reality and data for evaluation and validation of a more detailed fault model is seldom available. Moreover, modeling faults in this way also results in a minimum of fault modes, which gives a smaller model. This is beneficial since a smaller model simplifies several steps in model-based diagnosis, for example residual generation or fault isolation. In addition this is performed for simplicity. Thus extending the nominal model with additive fault signals is straightforward and easy. Nevertheless, the approach has shown to provide good results (Svärd and Nyberg, 2012).

The adopted approach is nonetheless general, and no assumptions are made regarding, for example, the time-behaviour of faults. For instance, the approach is able to handle multiplicative faults even though the fault signal is assumed to be additive. In essence, a multiplicative fault in $y_{p_{\mathrm{im}}}$ can be given by $y_{p_{\mathrm{im}}}=$ $\delta \cdot p_{\mathrm{im}}, \delta \neq 1$, which can be equivalently described by $\Delta_{y_{p_{\mathrm{im}}}}=p_{\mathrm{im}}(\delta-1)$.

\subsection{Model}

The model of the automotive diesel engine can be found in Appendix A. The model contains in total 46 equations, 43 unknown variables, 11 known variables, of which 4 are actuators and 7 sensors, and 9 faults. Of the 46 equations, 5 are differential equations and the rest algebraic equations. 
The model describes the gas-exchange system of the engine and is described in Wahlström and Eriksson (2011). The model relies on both fundamental first principle physics and gray-box modeling.

\subsubsection{Non-Linear Model Equations}

Due to the non-linear characteristics of the considered engine system, the model in Appendix A contains several non-linear functions. For instance, the function $\Psi_{\mathrm{th}}^{\gamma_{\mathrm{th}}}\left(\Pi_{\mathrm{th}}\right)$ found in equation $e_{7}$ is given by

$$
\Psi_{\mathrm{th}}\left(\Pi_{\mathrm{th}}\right)=\left\{\begin{array}{ll}
\Psi_{\mathrm{th}}^{*}\left(\Pi_{\mathrm{th}}\right) & \text { if } \Pi_{\mathrm{th}} \leq \Pi_{\mathrm{th}, \text { lin }} \\
\Psi_{\mathrm{th}}^{*}\left(\Pi_{\mathrm{th}, \mathrm{lin}}\right) \frac{1-\Pi_{\mathrm{th}}}{1-\Pi_{\mathrm{th}, \mathrm{lin}}} & \text { if } \Pi_{\mathrm{th}}>\Pi_{\mathrm{th}, \text { lin }}
\end{array},\right.
$$

where

$$
\Psi_{\mathrm{th}}^{*}\left(\Pi_{\mathrm{th}}\right)=\sqrt{\frac{2 \gamma_{\mathrm{th}}}{\gamma_{\mathrm{th}}-1}\left(\Pi_{\mathrm{th}}^{2 / \gamma_{\mathrm{th}}}-\Pi_{\mathrm{th}}^{1+1 / \gamma_{\mathrm{th}}}\right)},
$$

and $\Pi_{\text {th,lin }}$ and $\gamma_{\text {th }}$ are parameters. For more details, see Wahlström and Eriksson (2011). For notational simplicity, complicated non-linearities like (1) have

been denoted by functions named in analogy with $\Psi_{\mathrm{th}}^{\gamma_{\mathrm{th}}}\left(\Pi_{\mathrm{th}}\right)$. For instance, $f_{T_{\mathrm{e}} W_{\mathrm{f}}}\left(W_{\mathrm{f}}\right)$ in $e_{13}$ and $\eta_{\mathrm{tm}, \omega_{\mathrm{t}}}\left(\omega_{\mathrm{t}}\right)$ in $e_{18}$.

\section{Overview of Design Methodology}

This section presents an overview of the automated methodology used to design the FDI-system for the automotive diesel engine. The actual methods used in the different design stages are explained and discussed. Firstly, however, a brief description of the structure of the FDI-system is given. The notation used for describing the design methodology is summarized in Table 3.

\subsection{Structure of FDI-System}

The proposed FDI-system for the engine contains the sub-systems: residual generation, residual evaluation, and fault isolation, see Figure 2.

Measured signals, $y$, in this case from the actuators and sensors listed in Table 1, are used as input to the residual generation block. This block contains a set of residual generators, $R_{1}, R_{2}, \ldots, R_{n}$, each used to monitor a part of 
Table 3: Nomenclature for Design Methodology.

\begin{tabular}{ll}
\hline Symbol & Description \\
\hline$M$ & System model \\
$E$ & Set of equations \\
$X$ & Set of unknown variables \\
$D$ & Set of differentiated variables \\
$Y$ & Set of known variables \\
$F$ & Set of faults \\
$S$ & Subset of the equations in $E$ \\
$e$ & Equation \\
$\Delta$ & Fault \\
$y$ & Vector of known variables \\
$z$ & Vector of differentiated variables \\
$w$ & Vector of algebraic variables \\
$\mathcal{F}$ & Diagnosis requirement \\
$\mathcal{Y}$ & No-fault measurement data \\
$R$ & Residual generator \\
$r$ & Residual \\
$T$ & Residual evaluator \\
$\lambda$ & Test statistic \\
$J$ & Alarm threshold \\
$\mathcal{R}$ & Set of residual samples \\
$\theta$ & Matrix of residual distributions \\
$\theta^{\mathrm{NF}}$ & Matrix of no-fault residual distributions \\
$\alpha$ & Distribution weighting vector \\
$\mathcal{L}(\mid)$ & Likelihood function \\
\hline & \\
\hline
\end{tabular}

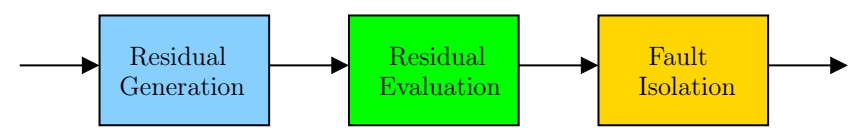

Measurements

Residuals

Detection Results

Isolation Results

Figure 2: Overview of the sub-systems in the FDI-system. 


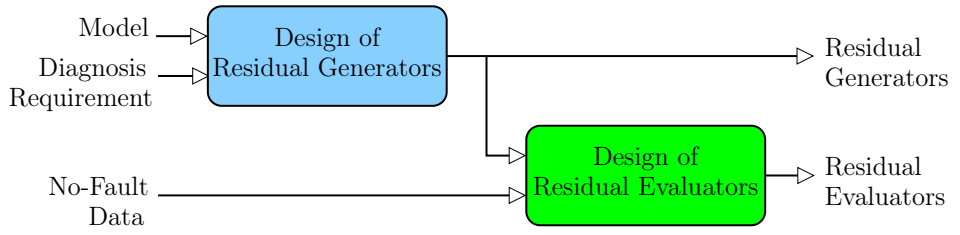

Figure 3: Overview of the two steps in the proposed design methodology.

the system. The output from the residual generation block is a set of residual signals, $r_{1}, r_{2}, \ldots, r_{n}$, with $r_{i}=R_{i}(y)$. The residual signals are used as input to the residual evaluation block, which contains a set of residual evaluators, $T_{1}, T_{2}, \ldots, T_{n}$. The aim of the residual evaluation is to detect changes in the residual signal behaviour caused by faults in the system. The output from the residual evaluation block is a set of binary fault detection signals, $d_{1}, d_{2}, \ldots, d_{n}$, with $d_{i}=T_{i}\left(r_{i}\right)$. Each $d_{i}$ indicates if a fault is present or not in the part of the system monitored by the corresponding residual generator $R_{i}$. The set of fault detection signals $d_{1}, d_{2}, \ldots, d_{n}$ is finally used as input to the fault isolation block, where they are used to isolate the detected fault(s).

\subsection{Automated Design Methodology}

An overview of the overall methodology used to design the residual generators and residual evaluators, is shown in Figure 3.

The design methodology depicted in Figure 3 have been developed with the aim to be automated to a high extent and requires limited human interaction. The methodology requires the following input:

- A model $M=(E, X, D, Y, F)$ of the system, where $E$ is a set of differentialalgebraic equations relating the unknown variables $X$, differentiated variables $D$, known variables $Y$, and fault variables $F$.

- A diagnosis requirement $\mathcal{F}$, given as a set of ordered fault pairs $\left(\Delta_{i}, \Delta_{j}\right) \in$ $F \times F$. The interpretation of a pair $\left(\Delta_{i}, \Delta_{j}\right) \in \mathcal{F}$ is that fault $\Delta_{i}$ should be detectable and isolable from fault $\Delta_{j}$.

- No-fault data $\mathcal{Y}$, given in the form of measurements of the variables contained in $Y$. 
Note that the requirement of full diagnosability of a fault $\Delta_{i}$ is described with the diagnosis requirement $\mathcal{F}$ by including $\left(\Delta_{i}, \Delta_{j}\right) \in \mathcal{F}$ for all $\Delta_{j} \in F$ such that $\Delta_{j} \neq \Delta_{i}$. For more details about detectability and isolability definitions and specifications, see Krysander and Frisk (2008).

The output is a set of residual generators $R_{1}, R_{2}, \ldots, R_{n}$, and a set of residual evaluators, $T_{1}, T_{2}, \ldots, T_{n}$. The specific methods used to design the residual generators and residual evaluators are described in the following sections. Design of the fault isolation subsystem is briefly discussed in Section 5.3.

\subsection{Residual Generation}

The method used to design the individual residual generators is described in Svärd and Nyberg (2010) and belongs to a class of methods referred to as sequential residual generation where the unknown variables are computed by solving one equation at a time in a sequence and a residual is then obtained by evaluating a redundant equation. These ideas were originally described in Staroswiecki and Declerck (1989) and similar approaches can be found in Cassar and Staroswiecki (1997); Staroswiecki (2002); Pulido and Alonso-González (2004); Ploix et al. (2005); Travé-Massuyès et al. (2006); Blanke et al. (2006).

This class of methods has shown to be successful for real applications (Dustegor et al., 2004; Izadi-Zamanabadi, 2002; Cocquempot et al., 1998), and also have the potential to be automated to a high extent (Svärd and Nyberg, 2012). The key property of the specific method described in Svärd and Nyberg (2010) is its ability to handle mixed causality, which greatly increases the possibility to detect and isolate faults in large-scale complex models. This issue is discussed and illustrated in Section 4.

In general, it is possible to create a large number of residual generators with the method from (Svärd and Nyberg, 2010) for complex models. Regarding implementation aspects such as complexity and computational load it is infeasible, or even impossible, to use all these residual generators in the FDI-system. In addition, it is often possible to meet the stated diagnosis requirement with a small subset of all residual generators. Therefore, the set of residual generators 


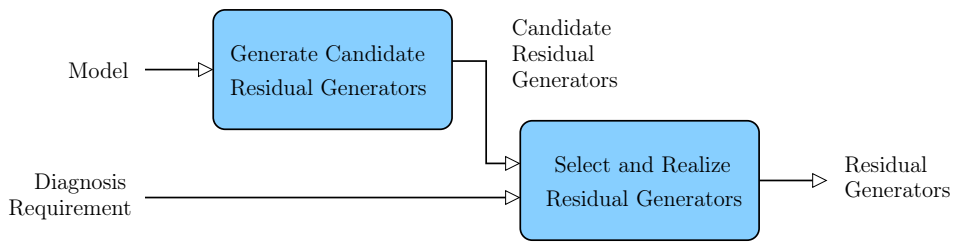

Figure 4: Method for design and selection of residual generators.

to be contained in the FDI-system is selected by means of a two-step approach, as also elaborated in Nyberg (1999); Krysander (2006); Nyberg and Krysander (2008), which is described next.

\subsubsection{Two-Step Approach}

Given the model $M$ of the system and the diagnosis requirement $\mathcal{F}$, the two steps illustrated in Figure 4 are conducted.

In the first step, a large set of candidate residual generators, in the form of subsets of the model equations, is found. This step is performed in an exhaustive manner, in the sense that all model equation subsets that can be used as input to the sequential residual generation method (Svärd and Nyberg, 2010) are found. For this particular method, it can be shown (Svärd and Nyberg, 2010) that candidate residual generators, by necessity, should be based on Minimal Structural Overdetermined (MSO) sets of equations since these are minimal sets of equations containing analytic redundancy. There exists efficient algorithms for finding all MSO sets, given a model, see, e.g., Krysander et al. (2008).

In general, all candidate residual generators found in the first step are not realizable, i.e., it is not possible to create residual generators from all found candidate residual generators with the considered method. Therefore, in the second step, a set of realizable candidate residual generators that fulfills the diagnosis requirement $\mathcal{F}$ are selected and the final set of residual generators $R_{1}, R_{2}, \ldots, R_{n}$ is created.

\subsubsection{Realizability of Candidate Residual Generators}

Realizability is a general property of a candidate residual generator, i.e., a set of equations, with respect to a given residual generation method, see Svärd 
(2012). In the context of the method (Svärd and Nyberg, 2010), a set of equations $S \subseteq E$ is said to be realizable if it can be written in the form

$$
\begin{aligned}
\dot{z} & =f\left(z, w_{1}, w_{2}, \ldots, w_{m}, y\right) \\
w_{1} & =g_{1}(z, y) \\
w_{2} & =g_{2}\left(z, w_{1}, \dot{w}_{1}, y\right) \\
& \vdots \\
w_{m} & =g_{m}\left(z, w_{1}, \dot{w}_{1}, w_{1}, \dot{w}_{2}, \ldots, w_{m-1}, \dot{w}_{m-1}, y\right)
\end{aligned}
$$

where $z$ is a vector of differentiated variables, $w_{i}, i=1,2, \ldots, m$, vectors of algebraic variables, and $y$ a vector of known variables. In addition, it is required that (2) is stable.

A sufficient condition for the ability to transform the equations in $S$ into the form (2), is the existence of a computation sequence for the unknown variables contained in $z$ and $w_{i}, i=1,2, \ldots, m$. The existence of a computation sequence depends naturally on the properties of the equations in $S$, but also on the causality assumption, i.e., regarding whether integral and/or derivative causality (Blanke et al., 2006) may be used to handle differential equations in the computation sequence. For further details, see Svärd and Nyberg (2010).

\subsubsection{Selection of Residual Generators}

Motivated by implementation aspects, it is desired in the second step to find a minimal cardinality set of realizable residual generators that fulfills the diagnosis requirement $\mathcal{F}$. If the number of the resulting candidate residual generators is large, which typically is the case for large-scale models such as the one considered in this work, the problem of finding such a minimal set of residual generators is hard, or even impossible, to solve optimally. However, by relaxing the minimal cardinality requirement, a near optimal solution to the selection problem can be efficiently computed by means of the greedy residual generator selection algorithm developed in Svärd et al. (2011a).

In the greedy selection algorithm, one of the candidate residual generators able to isolate most of the faults in the given diagnosis requirement $\mathcal{F}$ is selected. 


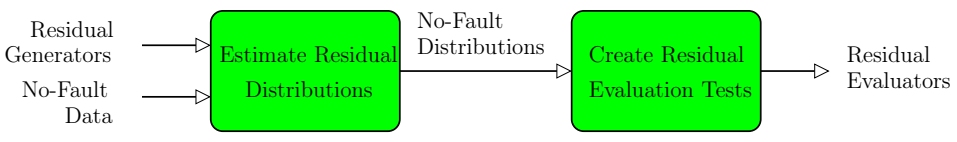

Figure 5: Method for design of residual evaluators.

This is added to the solution, if it is realizable otherwise it is removed from the remaining candidate residual generators. Then, in each iteration given the set of already selected and remaining candidate residual generators, a remaining candidate residual generator able to isolate most of the not already isolable faults in the given diagnosis requirement $\mathcal{F}$ is selected. This is added to the solution, if it is realizable otherwise it is removed from the remaining candidate residual generators. This procedure is repeated until $\mathcal{F}$ is fulfilled, or no useful candidate residual generators remain.

In addition to make the selection problem tractable, the greedy selection algorithm has some additional properties. Specifically, it can be shown (Svärd, 2012) that if, and only if, the given diagnosis requirement can be fulfilled for the given model with the method (Svärd and Nyberg, 2010), then the algorithm will provide a solution.

\subsection{Residual Evaluation}

The method used to design residual evaluators is described in Svärd et al. (2011b); Svärd (2012). The key property of this statistical and data-driven method is its ability to handle residuals whose stochastic behaviour vary with the current operating mode of the underlying system. The method is based on a comparison of the probability distribution of the residual, estimated online using current data, with a no-fault residual distribution. The no-fault distribution is based on a set of distributions estimated off-line using training data, and is continuously adapted to the current operating mode of the system.

The method used for design of residual evaluators is illustrated in Figure 5. Given a set of residual generators $R_{1}, R_{2}, \ldots, R_{n}$ and no-fault data $\mathcal{Y}$ in the form of measurements of the input to the residual generators, the residual generators are run and no-fault residual samples are created. By application of the method 
developed in Svärd (2012) which utilizes K-Means clustering (MacQueen, 1967; Lloyd, 1982), the set of no-fault residual samples is then used to estimate a set $\theta_{i}^{\mathrm{NF}}$ of $K$ no-fault distributions for each of the residuals $r_{1}, r_{2}, \ldots, r_{n}$, obtained as output from the residual generators $R_{1}, R_{2}, \ldots, R_{n}$.

\subsubsection{Residual Evaluators}

The obtained no-fault residual distributions are then used to create a residual evaluator $T_{i}$ for each of the residuals $r_{1}, r_{2}, \ldots, r_{n}$. The residual evaluator $T_{i}$, with the binary detection signal $d_{i}$ as output, comprises a fault detection test

$$
d_{i}=T_{i}\left(\mathcal{R}_{i}\right)= \begin{cases}1 & \text { if } \lambda_{i}\left(\mathcal{R}_{i}\right)>J_{i} \\ 0 & \text { else }\end{cases}
$$

where $\lambda_{i}$ is a test statistic, $\mathcal{R}_{i}$ is a set of discretized samples from residual $r_{i}$, and $J_{i}$ is a constant detection threshold.

The test statistic $\lambda_{i}$ in each fault detection test is designed with the method developed in Svärd et al. (2011b) and based on the Generalized Likelihood Ratio (GLR) test. Given a set $\mathcal{R}_{i}$ of samples of the residual $r_{i}$, and the matrix $\theta_{i}^{\mathrm{NF}}$ containing the estimated no-fault distributions of $r_{i}$, the test statistic is given by

$$
\lambda_{i}\left(\mathcal{R}_{i}\right)=-2 \log \frac{\max _{\alpha} \mathcal{L}\left(\alpha, \theta_{i}^{\mathrm{NF}} \mid \mathcal{R}_{i}\right)}{\max _{\alpha, \theta} \mathcal{L}\left(\alpha, \theta \mid \mathcal{R}_{i}\right)},
$$

where $\mathcal{L}\left(\alpha, \theta \mid \mathcal{R}_{i}\right)$ denotes the likelihood of the parameters $\alpha$ and $\theta$, given the residual samples in $\mathcal{R}_{i}$. The parameters $\alpha$ and $\theta$ fully specify the probability distribution of the samples in $\mathcal{R}_{i}$. In this sense, the quantity in the denominator of (4) corresponds to the most likely distribution of the samples in $\mathcal{R}_{i}$, and the quantity in the numerator to the most likely no-fault residual distribution.

\subsubsection{Maximum Likelihood Estimations}

In Svärd (2012), it is shown that an explicit solution to the maximum likelihood estimation (MLE) problem in the denominator of (4) can be obtained from the normalized histogram of the samples in $\mathcal{R}_{i}$. The MLE problem in the 
numerator however needs to be solved numerically. In order to enable implementation of the residual evaluators in an online environment subject to real-time constraints, this problem can be relaxed and posed as a constrained linear least square problem. This problem can be efficiently solved in real-time using methods based on convex optimization (Mattingley and Boyd, 2010). For technical details, see Svärd (2012).

\section{Design of Residual Generators}

As said in Section 1 it is by OBD-legislations required that emission critical faults in an automotive engine are detected and isolated. For the engine considered, all faults found in Table 2 are emission critical. In addition, if not accommodated in time, the faults in Table 2 may also lead to decreased safety, increased fuel consumption, decreased driveability, or even engine breakdown. The latter indeed reduces vehicle uptime. For a comprehensive discussion regarding diagnosis requirements for automotive engines in general, see Mohammadpour et al. (2012).

Motivated by this, it is required that all faults found in Table 2 can be detected and isolated from each other. Thus, the diagnosis requirement $\mathcal{F}$ for

the diesel engine consists of all unique pairwise combinations of the 9 faults in Table 2, i.e.,

$$
\mathcal{F}=\left\{\left(\Delta_{y_{p_{\mathrm{amb}}}}, \Delta_{y_{T_{\mathrm{amb}}}}\right),\left(\Delta_{y_{p_{\mathrm{amb}}}}, \Delta_{y_{p_{\mathrm{ic}}}}\right), \ldots,\right\}
$$

with $|\mathcal{F}|=9 \times 9-9=72$.

\subsection{Candidate Residual Generators}

The model of the engine given in Appendix A together with the diagnosis requirement $\mathcal{F}$, were used as input to a MATLAB implementation of the two-step residual generation methodology outlined in Section 3.3 and Figure 4.

In total 14, 242 candidate residual generators could be found for the engine model. These are based on 270 MSO sets, found using the algorithm described in Krysander et al. (2008). An MSO set by definition contains one more equation 
than unknown variables. Given an MSO set, a sequential residual generator is created by removing one equation and then finding a computation sequence for the unknown variables in the remaining just-determined set of equations. The number of candidate residual generators that can be created from a single MSO set thus equals the number of equations in the MSO set. This is the rationale behind the number of 14,242 candidate residual generators.

\subsection{Residual Generator Selection and Realization}

The algorithm (Svärd and Nyberg, 2010) for finding computation sequences for the candidate residual generators was configured to allow both integral and derivative causality, i.e., mixed causality, and also to use MAPLE as algebraic equation solving tool, see Section 3.3.2.

Using the greedy selection algorithm (Svärd et al., 2011a) described in 3.3.3, 8 residual generators, $R_{1}, R_{2}, \ldots, R_{8}$, were selected and realized. For instance, the residual generator $R_{3}$ has the form

$$
\begin{aligned}
\dot{\omega}_{\mathrm{t}} & =\frac{P_{\mathrm{t}} \eta_{m}-P_{\mathrm{c}}}{J_{\mathrm{t}} \omega_{\mathrm{t}}} \\
\dot{T}_{\mathrm{em}} & =\frac{R_{\mathrm{e}} T_{\mathrm{em}}}{p_{\mathrm{em}} V_{\mathrm{em}} c_{\mathrm{ve}}}\left(W_{\mathrm{in}} c_{\mathrm{ve}}\left(T_{\mathrm{em}, \mathrm{in}}-T_{\mathrm{em}}\right)+\right. \\
& \left.R_{\mathrm{e}}\left(T_{\mathrm{em}, \mathrm{in}} W_{\mathrm{in}}-T_{\mathrm{em}} W_{\mathrm{out}}\right)\right) \\
\dot{p}_{\mathrm{em}} & =\frac{R_{\mathrm{e}} T_{\mathrm{em}}}{V_{\mathrm{em}}}\left(W_{\mathrm{eo}}-W_{\mathrm{egr}}-W_{\mathrm{t}}+\Delta_{W_{\mathrm{em}}}\right) \\
& +\frac{R_{\mathrm{e}}}{V_{\mathrm{em}} c_{\mathrm{ve}}}\left(W_{\mathrm{in}} c_{\mathrm{ve}}\left(T_{\mathrm{em}, \mathrm{in}}-T_{\mathrm{em}}\right)\right. \\
& \left.+R_{\mathrm{e}}\left(T_{\mathrm{em}, \mathrm{in}} W_{\mathrm{in}}-T_{\mathrm{em}} W_{\mathrm{out}}\right)\right) \\
p_{\mathrm{amb}} & =y_{p_{\mathrm{amb}}} \\
p_{\mathrm{bc}} & =p_{\mathrm{amb}} \\
x_{\mathrm{vgt}} & =u_{x_{\mathrm{vgt}}} \\
\vdots & \\
T_{\mathrm{em}, \mathrm{in}} & =T_{\mathrm{amb}}+\left(T_{\mathrm{e}}-T_{\mathrm{amb}}\right) \exp \left(-\frac{h_{t o t} \pi d_{p i p e} l_{\text {pipe }} n_{\text {pipe }}}{W_{\mathrm{eo}} c_{\mathrm{pe}}}\right) \\
W_{\mathrm{egr}} & =\frac{\left(\dot{p}_{\mathrm{im}} V_{\mathrm{im}}-R_{\mathrm{a}} T_{\mathrm{im}} W_{\mathrm{th}}+W_{\mathrm{ei}} R_{\mathrm{a}} T_{\mathrm{im}}\right)}{R_{\mathrm{a}} T_{\mathrm{im}}} \\
\vdots & \\
P_{\mathrm{c}} & =\frac{W_{\mathrm{c}} c_{p a} T_{\mathrm{bc}}}{\eta_{\mathrm{c}}}\left(\Pi_{\mathrm{c}}^{1-1 / \gamma_{\mathrm{a}}}-1\right),
\end{aligned}
$$

with the residual equation $r=y_{p_{\mathrm{em}}}-p_{\mathrm{em}}$, corresponding to equation $e_{43}$ in Appendix A. 
Clearly, the structure of residual generator $R_{3}$ is in accordance with (2). Moreover, it is noted that residual generator $R_{3}$ exploits mixed causality. Integral causality is for example used in $(6 \mathrm{~b})$ when variable $T_{\mathrm{em}}$ is computed. Derivative causality is employed when variable $W_{\text {egr }}$ is computed in $(6 \mathrm{~h})$, since $\dot{p}_{\text {im }}$, the derivative of $p_{\text {im }}$, is used.

The use of derivative causality in general assumes that derivatives of known or previously computed variables can be computed or estimated. In this work, estimation of derivatives is done by appliance of a low-pass FIR-filter with coefficients calculated according to Vainio et al. (1997). This approach was used since it is simple and straightforward to implement, and gave sufficient results.

The use of integral causality presupposes that ordinary differential equations can be solved, which in general assumes that consistent initial conditions for the state-variables are available. There are 5 different state variables present in the set of selected residual generators: the intake manifold pressure $p_{\mathrm{im}}$, the exhaust manifold pressure $p_{\text {em }}$, intercooler pressure $p_{\text {ic }}$, the exhaust manifold temperature $T_{\mathrm{em}}$, and the turbine speed $\omega_{\mathrm{t}}$. As seen in Table 1 , the three pressures are measured directly. Thus, the values of the corresponding measured variables at the starting time instant are used as initial conditions for these variables, e.g., $p_{\mathrm{im}}\left(t_{0}\right)=y_{p_{\mathrm{im}}}\left(t_{0}\right)$. For the non-measured state-variable $T_{\mathrm{em}}$, the initial condition is set to the value of the measured inlet air temperature $y_{T_{\mathrm{im}}}$ at the starting time instant. The initial condition for the state-variable $\omega_{\mathrm{t}}$ is set to a constant nominal value.

\subsubsection{Fault Detectability}

Table 4 shows the fault signature matrix (FSM) for the 8 selected residual generators with respect to the faults in Table 2. In this context, the FSM contains an " $\mathrm{x}$ " in position $\left(R_{i}, \Delta_{x}\right)$ if the equation containing fault $\Delta_{x}$ is used in the computation sequence on which the residual generator $R_{i}$ is based. This should be interpreted as that residual generator $R_{i}$ may be sensitive to fault $\Delta_{x}$, meaning that it may respond to the fault. The sensitivity of residual generator $R_{i}$ to the fault $\Delta_{x}$ strongly depends on the properties of $R_{i}$, the size 
Table 4: Fault signature matrix for the 8 residual generators. An $x$ in position $(i, j)$ represents that fault mode $j$ may cause residual $i$ to raise an alarm.

\begin{tabular}{|c|c|c|c|c|c|c|c|c|c|}
\hline & ఫँ & ఫ & ${ }_{j}^{i}$ & $\frac{\xi}{j}$ & $\underset{j}{\stackrel{g}{5}}$ & $\underbrace{\sharp}$ & ${ }^{\frac{5}{4}}$ & 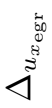 & $\underbrace{+\infty}$ \\
\hline$R_{1}$ & $\mathrm{X}$ & $\mathrm{X}$ & & $\mathrm{X}$ & $\mathrm{X}$ & $\mathrm{X}$ & & $\mathrm{x}$ & $\mathrm{X}$ \\
\hline$R_{2}$ & $\mathrm{X}$ & $\mathrm{X}$ & $\mathrm{X}$ & $\mathrm{X}$ & & $\mathrm{X}$ & $\mathrm{x}$ & $\mathrm{X}$ & $\mathrm{X}$ \\
\hline$R_{3}$ & $\mathrm{x}$ & $\mathrm{x}$ & $\mathrm{x}$ & $\mathrm{x}$ & $\mathrm{x}$ & $\mathrm{x}$ & $\mathrm{x}$ & & $\mathrm{X}$ \\
\hline$R_{4}$ & $\mathrm{x}$ & $\mathrm{x}$ & $\mathrm{x}$ & $\mathrm{x}$ & $\mathrm{x}$ & & $\mathrm{x}$ & $\mathrm{x}$ & $\mathrm{x}$ \\
\hline$R_{5}$ & $\mathrm{x}$ & & $\mathrm{x}$ & $\mathrm{x}$ & $\mathrm{x}$ & $\mathrm{x}$ & $\mathrm{x}$ & $\mathrm{x}$ & $\mathrm{x}$ \\
\hline$R_{6}$ & $\mathrm{X}$ & $\mathrm{X}$ & $\mathrm{X}$ & & $\mathrm{X}$ & $\mathrm{x}$ & $\mathrm{x}$ & $\mathrm{x}$ & $\mathrm{x}$ \\
\hline$R_{7}$ & $\mathrm{x}$ & $\mathrm{x}$ & $\mathrm{x}$ & $\mathrm{x}$ & $\mathrm{x}$ & $\mathrm{x}$ & & $\mathrm{x}$ & $\mathrm{x}$ \\
\hline$R_{8}$ & $\mathrm{x}$ & $\mathrm{x}$ & & $\mathrm{X}$ & $\mathrm{x}$ & $\mathrm{x}$ & $\mathrm{x}$ & $\mathrm{x}$ & $\mathrm{x}$ \\
\hline
\end{tabular}

and temporal properties of $\Delta_{x}$, and also on, for example, the current operating mode of the system. In order to verify that $R_{i}$ is indeed sensitive to $\Delta_{x}$, it is necessary to implement and run $R_{i}$ using representative data from relevant fault cases. This will be done in Section 6 .

Clearly, assuming that Table 4 reflects the fault sensitivity, there is more than one residual generator that is sensitive to each of the 9 considered faults and thus all 9 faults can, in theory, be detected.

\subsubsection{Fault Isolability}

In general, given a set of residual generators, a fault $\Delta_{x}$ is said to be isolable from a fault $\Delta_{y}$ if the set contains a residual generator that is sensitive to fault $\Delta_{x}$ but not to fault $\Delta_{y}$, see for example Svärd (2012). As seen in Table 4, all 8 residual generators may be sensitive to the faults $\Delta_{y_{p_{\mathrm{amb}}}}$ and $\Delta_{u_{x_{\mathrm{vgt}}}}$. This is also indicated in Table 5, which shows the resulting isolability matrix for the 8 selected residual generators. In Table 5 , for instance, the " $\mathrm{x}$ " in position $\left(\Delta_{y_{T_{\mathrm{amb}}}}, \Delta_{y_{p_{\mathrm{amb}}}}\right)$ denotes that fault $\Delta_{y_{T_{\mathrm{amb}}}}$ is not isolable from fault $\Delta_{y_{p_{\mathrm{amb}}}}$ using the residual generators $R_{1}, R_{2}, \ldots, R_{8}$.

Clearly, according to Table 5 , the diagnosis requirement $\mathcal{F}$ in $(5)$ is not met since, for example, $\Delta_{y_{T_{\mathrm{amb}}}}$ not is isolable from $\Delta_{y_{p_{\mathrm{im}}}}$. Nevertheless, due to the properties of the greedy selection algorithm discussed in Section 3.3, Table 5 shows the maximum attainable isolability for the engine model, given 
Table 5: Maximum attainable isolability properties for the engine model. An $x$ in position $(i, j)$ indicates that fault mode $i$ can not be isolated from fault mode $j$.

\begin{tabular}{|c|c|c|c|c|c|c|c|c|c|}
\hline & 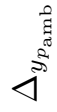 & 今a & $j^{0}$ & 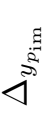 & $\begin{array}{l}\frac{8}{5} \\
\mathrm{D}^{\circ}\end{array}$ & 离 & 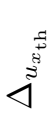 & & נ] \\
\hline$\Delta_{y_{p_{\mathrm{amb}}}}$ & $\mathrm{x}$ & & & & & & & & $\mathrm{x}$ \\
\hline$\Delta_{y_{T_{\mathrm{amb}} \mathrm{amb}}}$ & $\mathrm{x}$ & $\mathrm{x}$ & & & & & & & $\mathrm{x}$ \\
\hline$\Delta_{y_{p_{\mathrm{ic}}}}$ & $\mathrm{x}$ & & $\mathrm{x}$ & & & & & & $\mathrm{x}$ \\
\hline$\Delta_{y_{p_{\mathrm{im}}}}^{y_{\text {ic }}}$ & $\mathrm{x}$ & & & $\mathrm{x}$ & & & & & $\mathrm{x}$ \\
\hline$\Delta_{y_{T_{\mathrm{im}}}}$ & $\mathrm{x}$ & & & & $\mathrm{x}$ & & & & $\mathrm{x}$ \\
\hline$\Delta_{y_{p_{\mathrm{em}}}}$ & $\mathrm{x}$ & & & & & $\mathrm{x}$ & & & $\mathrm{x}$ \\
\hline$\Delta_{u_{x_{\text {th }}}}$ & $\mathrm{x}$ & & & & & & $\mathrm{x}$ & & $\mathrm{x}$ \\
\hline$\Delta_{u_{x_{\mathrm{egr}}}}$ & $\mathrm{x}$ & & & & & & & $\mathrm{x}$ & $\mathrm{x}$ \\
\hline$\Delta_{u_{x_{x}}}$ & $\mathrm{x}$ & & & & & & & & $\mathrm{x}$ \\
\hline
\end{tabular}

the method for residual generation considered in this work. The cardinality of the set of selected residual generators may however not be minimal. See Svärd (2012) for more details.

\subsection{Properties of Selected Residual Generators}

Some additional properties for the 8 selected residual generators can be found in Table 6 . The first column in Table 6 shows which residual equation the corresponding residual generator uses. It can be noted that a majority of the 8 residual generators use either equation $e_{39}$, or equation $e_{41}$, as residual equation, corresponding to $r=y_{p_{\mathrm{im}}}-p_{\mathrm{im}}$ and $r=y_{p_{\mathrm{em}}}-p_{\mathrm{em}}$, respectively. This is a direct consequence of that the greedy selection algorithm was supplemented with an additional heuristic in order to make the final deployment of the residual generators as simple as possible. In those cases when the greedy heuristic, described in Section 3.3.3, identified more than one candidate, the algorithm was configured to prefer small candidate residual generators, in terms of the number of equations, before large candidate residual generators, and also to prefer candidate residual generators using sensor equations, i.e., $e_{36}, e_{37}, \ldots, e_{41}$, as residuals.

Columns 2 and 3 in Table 6 show if the corresponding residual generator uses integral causality (IC) and/or derivative causality (DC), respectively. Clearly, 5 
Table 6: Properties of the selected residual generators.

\begin{tabular}{cccccc} 
& Residual & IC & DC & \#Equations & \#Inputs \\
\hline$R_{1}$ & $e_{41}$ & $\mathrm{x}$ & $\mathrm{x}$ & $42(5)$ & 9 \\
$R_{2}$ & $e_{7}$ & $\mathrm{x}$ & $\mathrm{x}$ & $43(5)$ & 10 \\
$R_{3}$ & $e_{41}$ & $\mathrm{x}$ & $\mathrm{x}$ & $43(4)$ & 10 \\
$R_{4}$ & $e_{39}$ & $\mathrm{x}$ & & $44(4)$ & 10 \\
$R_{5}$ & $e_{39}$ & $\mathrm{x}$ & $\mathrm{x}$ & $44(4)$ & 10 \\
$R_{6}$ & $e_{41}$ & $\mathrm{x}$ & & $44(4)$ & 10 \\
$R_{7}$ & $e_{41}$ & $\mathrm{x}$ & & $41(3)$ & 10 \\
$R_{8}$ & $e_{39}$ & $\mathrm{x}$ & $\mathrm{x}$ & $43(5)$ & 10
\end{tabular}

out of 8 residual generators employ mixed causality. Column 4 shows the number of equations contained in the computation sequence on which the corresponding residual generator is based, and the value in parenthesis how many of those equations that are differential equations. Recalling that the model contains in total 46 equations, of which 5 are differential equations, it can be concluded that all residual generators use a substantial part of the complete model in spite of the above mentioned heuristic. Column 5 in Table 6 shows how many of the 11 available signals in Table 1 that each residual generator uses as input. Clearly, all the residual generators use most of the available signals.

Columns 4 and 5 explain why most of the 8 selected residual generators may be sensitive to most of the 12 faults, as illustrated in Table 4 . In fact, this property holds for all candidate residual generators which on average use about 40 equations, and is a direct consequence of the properties of the automotive engine system. More specific, the engine system contains many physical interconnections such as for instance the shaft between the turbine and the compressor, which connects the intake and the exhaust parts of the engine, see Figure 1. This leads to a model with coupled equations, in the sense that there are sets of equations containing the same set of unknown variables. This implies that a fault affecting one of these equations influences a large amount of the other model equations. This, in combination with the relatively small number of sensors, makes fault decoupling non-trivial and results in the situation shown in Table 4. 
Table 7: Isolability matrix when using only residuals with integral causality. Compare with Table 5 to see the loss of isolability performance when forcing integral causality.

\begin{tabular}{|c|c|c|c|c|c|c|c|c|c|}
\hline & 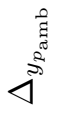 & 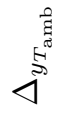 & $\underset{j}{i}$ & $\frac{a}{\sigma^{2}}$ & $\frac{a}{4}$ & ${ }^{\sharp}$ & 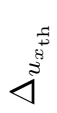 & 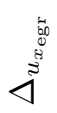 & تj \\
\hline$\Delta_{y_{p_{\mathrm{amb}}}}$ & $\mathrm{x}$ & $\mathrm{x}$ & & & $\mathrm{x}$ & & & $\mathrm{x}$ & $\mathrm{X}$ \\
\hline$\Delta_{y_{T_{\mathrm{amb}}}}$ & $\mathrm{x}$ & $\mathrm{x}$ & & & $\mathrm{x}$ & & & $\mathrm{x}$ & $\mathrm{x}$ \\
\hline$\Delta_{y_{p_{\text {ic }}}}$ & $\mathrm{x}$ & $\mathrm{x}$ & $\mathrm{x}$ & & $\mathrm{x}$ & & & $\mathrm{x}$ & $\mathrm{x}$ \\
\hline$\Delta_{y_{p_{\text {im }}}}$ & $\mathrm{x}$ & $\mathrm{x}$ & & $\mathrm{x}$ & $\mathrm{x}$ & & & $\mathrm{x}$ & $\mathrm{x}$ \\
\hline$\Delta_{y_{T_{\mathrm{im}}}}$ & $\mathrm{x}$ & $\mathrm{x}$ & & & $\mathrm{x}$ & & & $\mathrm{x}$ & $\mathrm{x}$ \\
\hline$\Delta_{y_{p_{\mathrm{em}}}}$ & $\mathrm{x}$ & $\mathrm{x}$ & & & $\mathrm{x}$ & $\mathrm{x}$ & & $\mathrm{x}$ & $\mathrm{x}$ \\
\hline$\Delta_{u_{x_{\mathrm{th}}}}$ & $\mathrm{x}$ & $\mathrm{x}$ & & & $\mathrm{x}$ & & $\mathrm{x}$ & $\mathrm{x}$ & $\mathrm{x}$ \\
\hline$\Delta_{u_{x_{\mathrm{egr}}}}$ & $\mathrm{x}$ & $\mathrm{x}$ & & & $\mathrm{x}$ & & & $\mathrm{x}$ & $\mathrm{x}$ \\
\hline$\Delta_{u_{x_{\mathrm{vgt}}}}$ & $\mathrm{x}$ & $\mathrm{x}$ & & & $\mathrm{x}$ & & & $\mathrm{x}$ & $\mathrm{x}$ \\
\hline
\end{tabular}

\subsection{Comments on Realizability}

The results presented above were obtained using mixed causality, i.e., computation sequences with both integral and derivative causality were allowed. For comparison, the algorithm (Svärd and Nyberg, 2010) for finding computation sequences was also configured to use solely integral and derivative causality. For the case with derivative causality, no realizable candidate residual generators were found. In the integral causality case, a set of 4 residual generators was selected. In fact, two of these residual generators were also found when adopting mixed causality and can be found as $R_{6}$ and $R_{7}$ in Table 6 .

Before termination, the greedy selection algorithm discarded in total 4,739 of the 14,242 candidate residual generators for not being realizable in the mixed causality case, and 7,133 candidates in the integral causality case. The corresponding numbers in terms of MSO sets are 91 and 135, respectively, out of 270. In the derivative causality case, all candidate residual generators were discarded due to non-realizability. It can be concluded that mixed causality improves realizability, in the sense that considerably more candidate residual generators can be realized. This implies that more faults can be isolated. This can be seen by comparing Table 5 with Table 7 , which shows the resulting isolability matrix when using only integral causality.

The large amount of discarded candidate residual generators, independent 
on the causality assumption, is due to that no computation sequence can be found for these candidate residual generators. This in turn is to a large extent caused by non-invertible non-linear functions in the model. To illustrate this aspect, consider the equation

$$
e_{7}: \quad W_{\mathrm{th}}=\frac{p_{\mathrm{ic}} A_{\mathrm{th}, \max }}{\sqrt{T_{\mathrm{im}} R_{\mathrm{a}}}} \Psi_{\mathrm{th}}^{\gamma_{\mathrm{th}}}\left(\Pi_{\mathrm{th}}\right) f_{\mathrm{th}}\left(x_{\mathrm{th}}\right),
$$

where $W_{\mathrm{th}}, p_{\mathrm{ic}}, T_{\mathrm{im}}, \Pi_{\mathrm{th}}$, and $x_{\mathrm{th}}$ are unknown variables, $A_{\mathrm{th}, \max }$ and $R_{\mathrm{a}}$ are parameters, and $\Psi_{\mathrm{th}}^{\gamma_{\mathrm{th}}}(\cdot)$ and $f_{\mathrm{th}}(\cdot)$ are non-linear functions, with $\Psi_{\mathrm{th}}^{\gamma_{\mathrm{th}}}\left(\Pi_{\mathrm{th}}\right)$ given by (1). Clearly, the function $\Psi_{\mathrm{th}}^{\gamma_{\mathrm{th}}}\left(\Pi_{\mathrm{th}}\right)$ is not invertible with respect to $\Pi_{\mathrm{th}}$ which implies that the variable $\Pi_{\mathrm{th}}$ can not be computed from the equation $e_{7}$. The same holds for the variable $x_{\mathrm{th}}$, since the function $f_{\mathrm{th}}(\cdot)$ is non-invertible with respect to $x_{\mathrm{th}}$. This implies that only the variables $W_{\mathrm{th}}, p_{\mathrm{ic}}$, and $T_{\mathrm{im}}$, can be computed from equation $e_{7}$. Most of the equations in the diesel engine model exhibit this property, and this substantially limits how unknown variables in the model can be computed, which in turn explains the large amount of nonrealizable, and thus discarded, candidate residual generators.

\subsubsection{Stability Analysis}

In comparison, only a fraction of the discarded candidate residual generators were discarded due to not being stable. Nevertheless, the stability analysis is an important part of the realization algorithm since stability is an important property in order to guarantee good dynamical behaviour of residual generators. In fact, the considered diesel engine system exhibits a non-minimum phase behaviour, see Wahlström and Eriksson (2011) for an analysis regarding this, which imply that there indeed are unstable candidate residual generators.

For sake of simplicity, the stability analysis is based on linearization. This is due to the fact that the stability analysis needs to be performed in an automated manner. In each of 20 different equilibrium points, the non-linear residual generator obtained from the series of computations described by the corresponding computation sequence, is first linearized. If any of the eigenvalues of the linearized residual generator is greater or equal to zero in any of the 20 equilibrium points, the residual generator is discarded. 


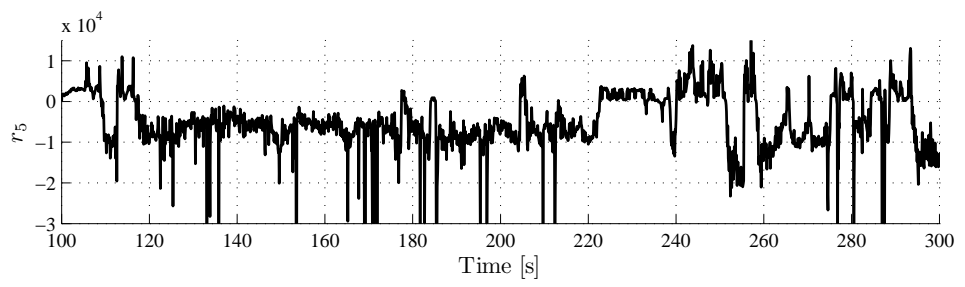

Figure 6: A subset of no-fault samples from residual $r_{5}$.

The 20 equilibrium points correspond to stationary operating points of the engine, parameterized by the injected fuel amount, $u_{\delta}$, and engine speed, $u_{n_{e}}$. The linearization is done by finite difference approximation. Although the adopted stability analysis approach is simple, it is able to discard the residual generators that were observed to be unable to use due to instability. This has been verified through extensive experimental evaluations.

\section{Design of Residual Evaluators}

As mentioned in Section 3.4, the first step in the residual evaluator design method is to estimate the probability distributions of the residuals $r_{1}, r_{2}, \ldots, r_{8}$ obtained as output from the residual generators $R_{1}, R_{2}, \ldots, R_{8}$, given the nofault data set $\mathcal{Y}$.

\subsection{Estimation of No-Fault Residual Distributions}

To capture the behaviour of the residuals in a variety of the operating modes of the diesel engine system, the no-fault data set $\mathcal{Y}$ was formed from two data sets of different characteristics. The first data set is about half an hour long and contains engine test-bed measurements from a World Harmonized Transient Cycle (WHTC) test cycle. The second data set is approximately 2 hours long and contains measurements from a part of a test drive in the south of Sweden, including both city and high-way driving. To reduce the risk of over-fit, the data sets were split into an estimation data set and a validation data set, of equal size. The data was sampled at a rate of $100 \mathrm{~Hz}$, and consequently the estimation and validation data sets contain approximately 450,000 samples, each. 

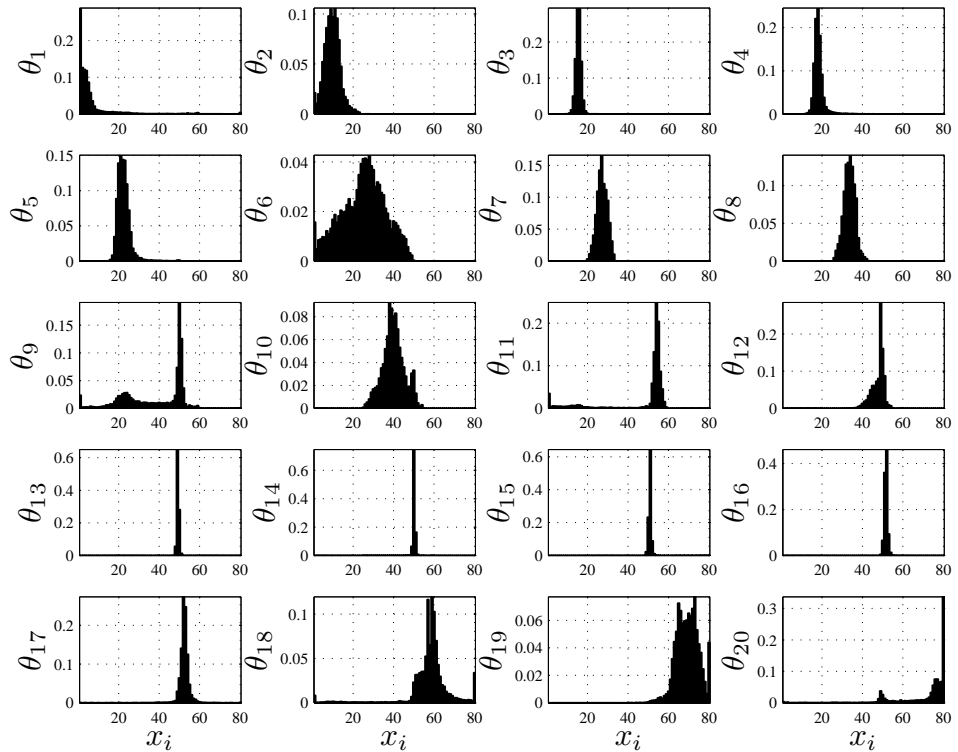

Figure 7: The set of 20 estimated no-fault distributions for residual $r_{5}$.

The 8 residual generators were run off-line using the measurements in $\mathcal{Y}$ as input to obtain no-fault residual samples. A set of samples from residuals $r_{5}$ is shown in Figure 6. Note the non-ideal behaviour of the residual caused by uncertainties, mainly model errors of time-varying nature and magnitude, mentioned in Section 1.

Using a MATLAB implementation of the algorithm in Svärd (2012), a set $\theta^{\mathrm{NF}}$ of $K=20$ probability density functions were estimated for each residual, see Section 3.4. Figure 7 shows the 20 estimated no-fault residual distributions for the residual $r_{5}$ obtained as output from residual generator $R_{5}$.

For this application, 20 distributions per residual is a good trade-off between model fit and complexity since the gain in model fit obtained when choosing a higher number is marginal in comparison with the corresponding increase in computational effort. This is illustrated in Figure 8, which shows the model fit in the form of the log-likelihood $\ell\left(\theta^{\mathrm{NF}} \mid \mathcal{Y}\right)$ of the distributions in $\theta^{\mathrm{NF}}$ given the no-fault data $\mathcal{Y}$. The quantity shown in Figure 8 is the averaged model fit for all 8 residuals, evaluated for different number of distributions and for both the 


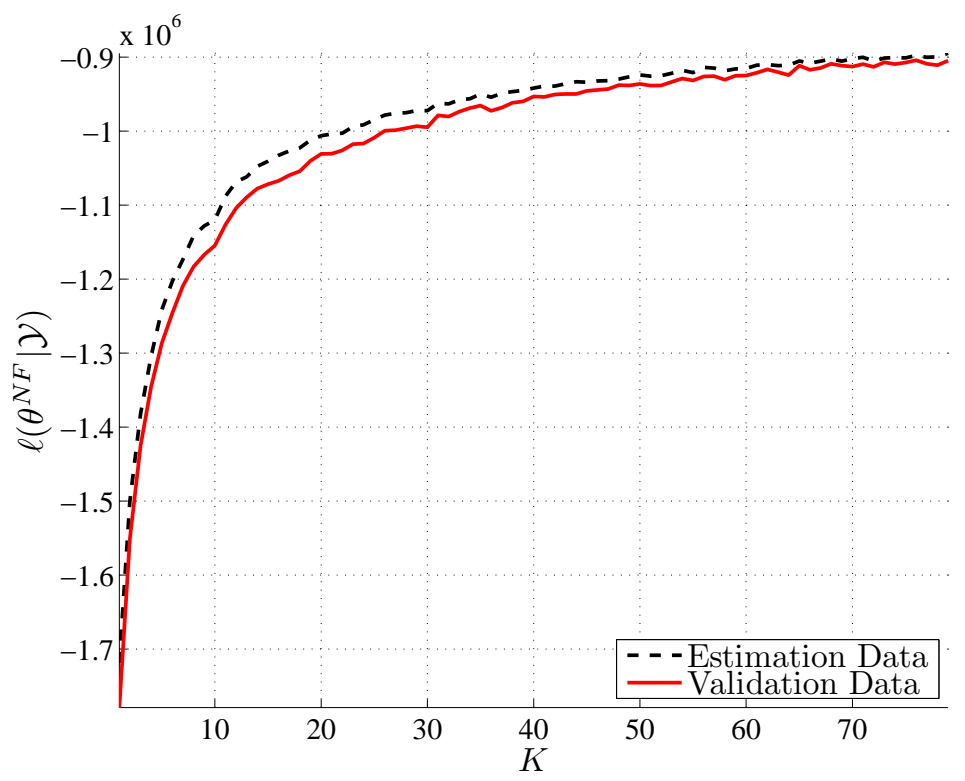

Figure 8: Fit of the set of estimated no-fault distributions for different values of $K$, i.e., for different number of distributions in the set, to the estimation and validation data sets. The figure shows the average of the fit for all 8 residuals.

estimation and validation data.

\subsection{Residual Evaluators}

For each of the residuals $r_{1}, r_{2}, \ldots, r_{8}$, a residual evaluator $T_{i}$ in the form (3) was created. The sampling of residual values for the sets $\mathcal{R}_{i}, i=1,2, \ldots, 8$, was done by means of a sliding window. The number of samples in each sliding window was chosen to be 1024 . The choice of this number is a trade-off between detection performance and computational complexity. For a thorough discussion of this issue, see Svärd (2012).

To solve the relaxed version of MLE problem in the numerator of (4), see Section 3.4.2, a tailored solver was generated using the software tool CVXGEN (Mattingley and Boyd, 2012). The detection thresholds $J_{i}, i=1,2, \ldots, 8$, were computed in order to give a probability of false detection of $1 \%$, by using the validation data set used in Section 5.1. 


\subsection{Fault Isolation Strategy}

As illustrated in Figure 2, the binary fault detection signals based on the residual evaluators (3), are used as input to the fault isolation block. This section briefly describes the strategy used for fault isolation.

Due to the issue regarding fault sensitivity discussed in Section 4.2.1, and since the complete behaviour of the no-fault residuals are not captured by the estimated no-fault distributions, the statistical power of the fault detection tests in (3) are not ideal. That is, the probability for detection is not one for all faults, in all situations, and the probability for false detections is not always zero. To take this into account, the fault isolation scheme is configured to interpret an " $\mathrm{x}$ " in a certain row of the FSM in Table 4 as if the test in the corresponding residual evaluator may respond, if the corresponding fault occurs. Consequently, no conclusion is drawn if a residual evaluator does not trigger any alarms, see Nyberg (1999).

Given a set of residual evaluators generating alarms, i.e., non-zero detection signals $d_{i}$, the fault signatures of the corresponding residuals are matched using the FSM in Table 4 . For an example, if only $d_{1}=1$, the row corresponding to $R_{1}$ in Table 4 is considered and it is concluded that either of the faults $\Delta_{y_{p_{\mathrm{amb}}}}, \Delta_{y_{T_{\mathrm{amb}}}}, \Delta_{y_{p_{\mathrm{im}}}}, \Delta_{y_{T_{\mathrm{im}}}}, \Delta_{y_{p_{\mathrm{em}}}}, \Delta_{u_{x_{\mathrm{egr}}}}$, and $\Delta_{u_{x_{\mathrm{vgt}}}}$, may be present in the system. If also the detection signals $d_{2}, d_{3}, d_{4}, d_{5}, d_{7}$, and $d_{8}$, are non-

zero, it is concluded that either of the faults $\Delta_{y_{p_{\mathrm{im}}}}, \Delta_{y_{p_{\mathrm{amb}}}}$, and $\Delta_{u_{x_{\mathrm{vgt}}}}$, may be present. This is in accordance with standard consistency-based diagnosis, see, e.g., de Kleer and Williams (1987); Reiter (1987); Greiner et al. (1989).

\section{Experimental Evaluation}

This section presents an experimental evaluation of the designed FDI-system. The evaluation consists of two parts, with different purposes. The first part, presented in Section 6.1, focuses on the fault detection performance of the individual residual generators and residual evaluators, whereas the second part, presented in Section 6.2, focuses on the detection and isolation performance of the complete FDI-system. 


\subsection{Fault Detection Performance}

The purpose of this part of the evaluation is to investigate the fault detection performance of the individual fault detection tests, comprised of the residual generators along with their corresponding residual evaluators.

\subsubsection{Metrics}

The fault detection performance is studied by means of the statistical power of the fault detection tests, for different sizes of the considered faults in Table 2. To quantify the power of a test, the power function (Casella and Berger, 2001) will be used. In this context, the power function for the fault detection test (3) for residual $r_{i}$ is defined as

$$
\beta_{i}(\delta)=\operatorname{Pr}\left(d_{i}=1 \mid \delta\right)=\operatorname{Pr}\left(\lambda_{i}\left(\mathcal{R}_{i}\right)>J_{i} \mid \delta\right),
$$

where $\lambda_{i}$ is the test statistic, $\mathcal{R}_{i}$ a set of samples from residual $r_{i}, J_{i}$ is the detection threshold, and $\delta$ is a fixed fault size. In the no-fault case, i.e., when $\delta$ corresponds to a fault of size zero, the power function (7) gives the probability of false detection, or Type I error (Casella and Berger, 2001). Otherwise, the power function gives the probability of detection for fixed $\delta$, or equivalently the probability of missed detection or Type II error, by $1-\beta_{i}(\delta)$.

In order to obtain a scalar metric for the detection performance of a specific detection test with respect to a set $D$ of different fault sizes, the quantity

$$
\frac{1}{|D|} \sum_{\delta \in D} \beta_{i}(\delta),
$$

will also be considered, where $\beta_{i}(\delta)$ is the power function for detection test $i$. The quantity (8) in some sense reflects the average detection performance of the detection test. It may be noted that for an ideal test, i.e., whose probability for detection is one for all fault sizes, the quantity (8) is equal to one.

\subsubsection{Setup}

In total 5 data sets were used in the evaluation. The data is not the same as the data described in Section 5. Each data set contains measurements collected during a drive on the Swedish west coast. The data sets contain measurements 
from approximately 2.5 hours of driving, and includes both high-way and city driving under different conditions.

The considered fault type is a gain fault. In the case of, for example, sensor fault $\Delta_{y_{p_{\mathrm{amb}}}}$, this means that the sensor signal $y_{p_{\mathrm{amb}}}$ fed to the residual generators is $y_{p_{\mathrm{amb}}}=\delta \cdot p_{\mathrm{amb}}$ where $\delta \neq 1$ indicates a fault. The gain faults were implemented off-line by modification of the corresponding sensor or actuator measurement $=$ signals.

Another alternative would have been to implement faults on-line, i.e., manipulations of hardware and/or the software during operation of the system. However, this approach is associated with a number of practical problems. One is that the fault injection typically results in a detection by the already existing on-board diagnosis system. This detection is then followed by a system shut-off or a compensation of the fault. Alternatively, the existing diagnosis system does not detect the fault but in that case, the injected fault may result in potential damage of the system. Therefore, to be able to cover the whole set of considered faults in a consistent way, the off-line approach was chosen. It should however be mentioned, that the considered set of faults is only an example, used primarily to illustrate the methodology proposed in the paper. In a real industrial situation, an evaluation of a diagnosis system by means of fault injection, should be based on a carefully selected fault set, where all issues like representativeness of real faults, cost of implementation, and practical limitation, should be weighted together.

\subsubsection{Behaviours of Residuals and Test Statistics}

Before presenting quantitative results by means of the metrics (7) and (8) some qualitative results are presented in order to provide some insight of the properties of the residuals and test statistics on which the fault detection tests are based.

Figure 9 shows the residuals $r_{1}, r_{2}, \ldots, r_{8}$ and test statistics $\lambda_{1}, \lambda_{2}, \ldots, \lambda_{8}$ when fault $\Delta_{y_{p_{\text {ic }}}}$ of size $\delta=1.2$ is abruptly injected at time $t=700 \mathrm{~s}$. Figure 10 shows the residuals and test statistics when fault $\Delta_{u_{x_{\mathrm{th}}}}$ of size $\delta=0.3$ is injected 
at time $t=700 \mathrm{~s}$.

First of all, it is noted that the residuals in Figures 9a and 10a are all non-zero in both the no-fault and fault cases. In addition, all residuals exhibit non-stationary behaviours. It is clear that a conventional residual evaluation approach by means of for example constant thresholding would not be sufficient for these residuals. Moreover, consider for instance residual $r_{5}$ in Figure 10a whose response to the fault is quite subtle, in the sense that the behaviour of the residual before and after the fault injection is similar. Nevertheless, the test statistic $\lambda_{5}$ clearly indicates the presence of a fault.

According to the FSM in Table 4, residuals $r_{1}$ and $r_{8}$ may be sensitive to fault $\Delta_{y_{p_{\text {ic }}}}$. This is hard to deduce from Figure 9a, but evident in Figure 9b since all test statistics but $\lambda_{1}$ and $\lambda_{8}$ respond clearly to the injected fault. However, the test statistic $\lambda_{7}$ do not cross the detection threshold. It is noted that this indeed corresponds to a typical situation and is taken into account in the fault isolation scheme, see Section 5.3. It may be noted that a traditional column matching approach (Gertler, 1998) is not sufficient for this, typical, case.

For the fault $\Delta_{u_{x_{\mathrm{th}}}}$, Table 4 states that residuals $r_{1}$ and $r_{7}$ should not be sensitive to the fault. Again, this is hard to tell from Figure 10a. However, Figure $10 \mathrm{~b}$ clearly shows that test statistics $\lambda_{1}$ and $\lambda_{7}$ do not respond to the fault. This can be seen in Figure 10b where the response from the test statistic $\lambda_{3}$ is weak and it only barely crosses the detection threshold. The responses from test statistics $\lambda_{2}$ and $\lambda_{8}$ are even weaker and they do not cross the detection thresholds at all. This will be discussed in Sections 6.1.4 and 6.2.

\subsubsection{Results and Comments}

Table 8 shows the quantity (8) for the fault detection test based on the residual evaluators $T_{1}, T_{2}, \ldots, T_{8}$ and all faults in Table 2. Entries close to zero, specifically $\leq 0.02$, have been marked bold. The right most column gives the average of each row, with the bold entries removed, and the same holds for the last row, but instead for the columns. Figure 11 explicitly shows the

estimated power functions $\beta_{1}, \beta_{2}, \ldots, \beta_{8}$ for the faults $\Delta_{y_{p_{\text {ic }}}}$ and $\Delta_{u_{x_{\mathrm{th}}}}$. The 

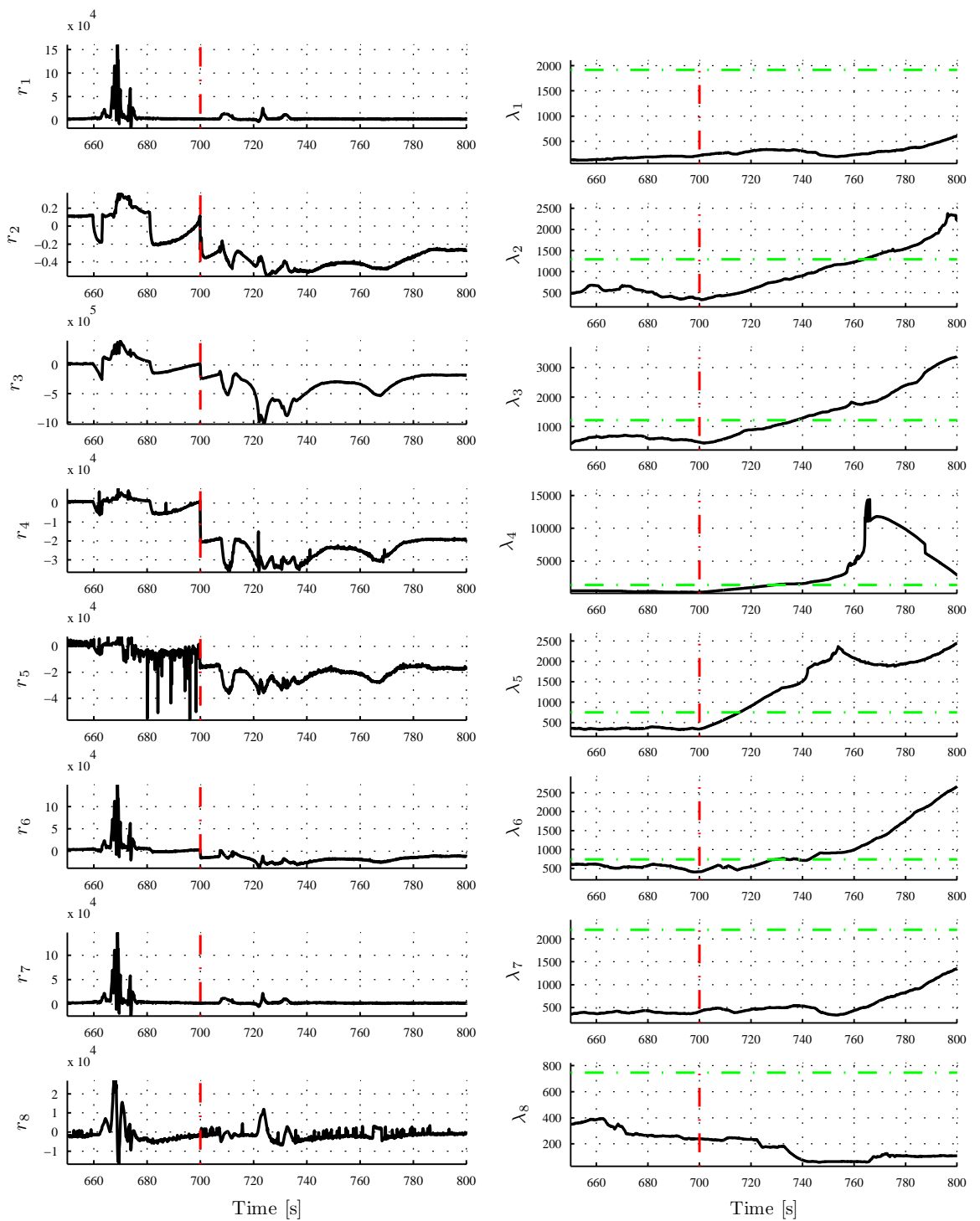

(a) Residuals

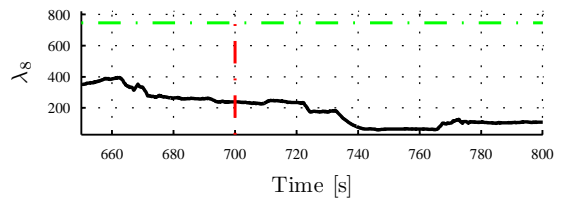

(b) Test Statistics

Figure 9: Residuals $r_{1}, r_{2}, \ldots, r_{8}$ and test statistics $\lambda_{1}, \lambda_{2}, \ldots, \lambda_{8}$ when fault $\Delta_{y_{p_{\text {ic }}}}$ injected at time $t=700 \mathrm{~s}$. 

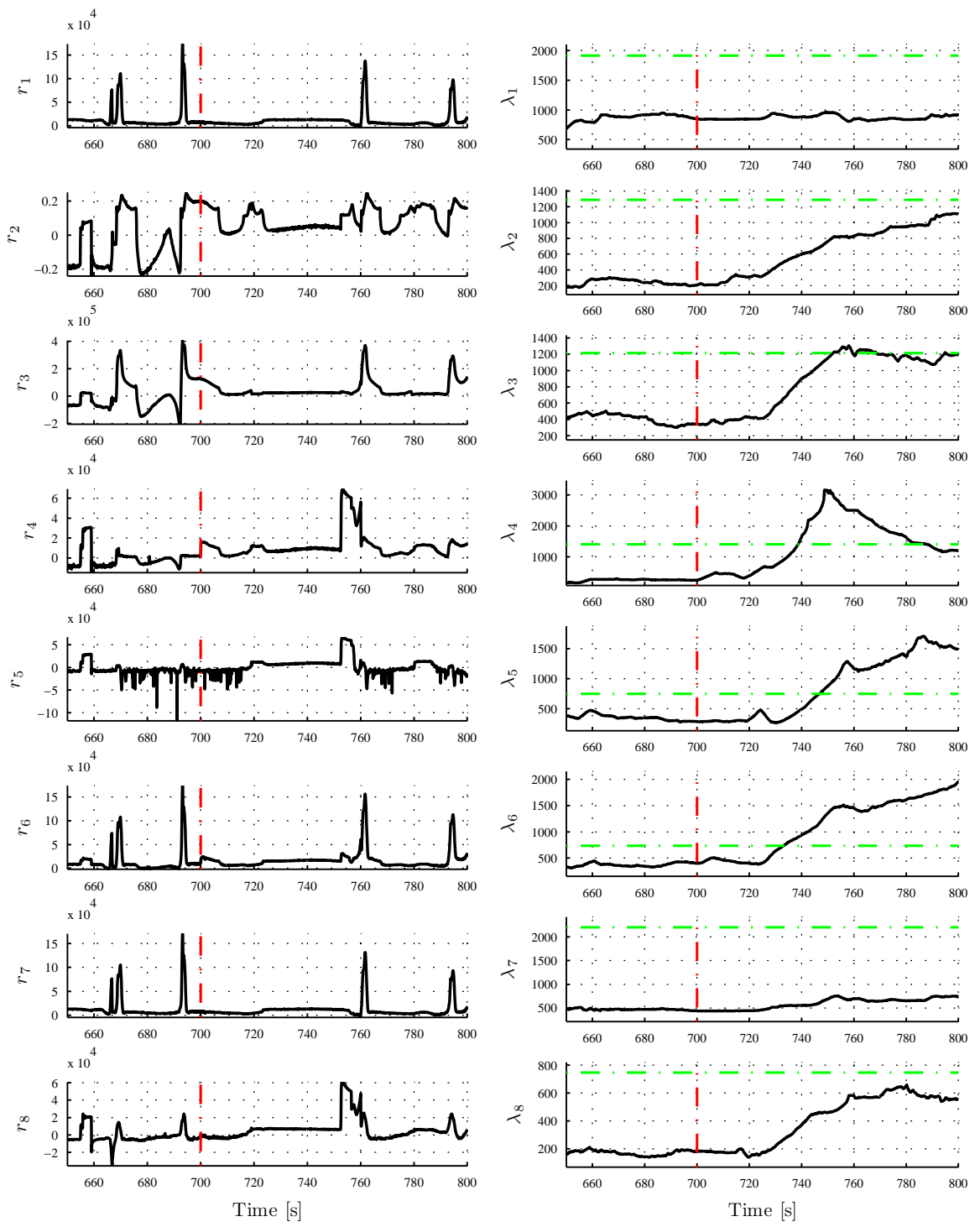

(a) Residuals

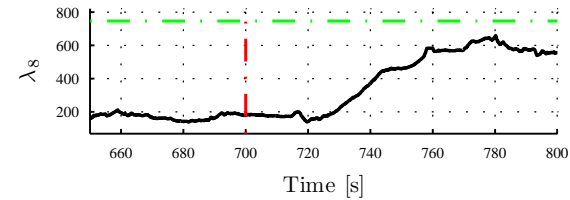

(b) Test Statistics

Figure 10: Residuals $r_{1}, r_{2}, \ldots, r_{8}$ and test statistics $\lambda_{1}, \lambda_{2}, \ldots, \lambda_{8}$ when fault $\Delta_{u_{x_{\text {th }}}}$ injected at time $t=700 \mathrm{~s}$. 
Table 8: Averaged power for all tests and all faults.

\begin{tabular}{|c|c|c|c|c|c|c|c|c|c|c|}
\hline & 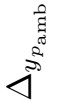 & 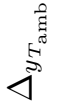 & $\underbrace{0}_{i}$ & $\underset{j}{\stackrel{5}{5}}$ & 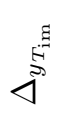 & 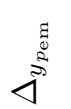 & 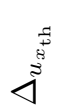 & 范 & נ] & \\
\hline$T_{1}$ & .01 & .03 & 0 & .17 & .18 & .74 & .01 & .05 & .11 & .22 \\
\hline$T_{2}$ & .38 & .06 & .62 & .32 & .01 & .38 & .09 & .01 & .11 & .28 \\
\hline$T_{3}$ & .07 & .05 & .75 & .40 & .07 & .25 & .06 & .01 & .16 & .23 \\
\hline$T_{4}$ & .01 & 0 & .83 & .47 & .02 & 0 & .11 & 0 & .02 & .49 \\
\hline$T_{5}$ & .01 & 0 & .75 & .53 & .12 & .44 & .16 & .01 & 0 & .41 \\
\hline$T_{6}$ & .09 & .10 & .81 & .01 & .40 & .86 & .11 & .06 & .34 & .35 \\
\hline$T_{7}$ & .01 & .04 & 0 & .19 & .13 & .39 & .01 & .07 & .12 & .16 \\
\hline$T_{8}$ & .65 & .41 & .02 & .45 & .59 & .84 & .12 & .05 & .33 & .43 \\
\hline & .31 & .12 & .76 & .36 & .26 & .56 & .11 & .07 & .20 & \\
\hline
\end{tabular}

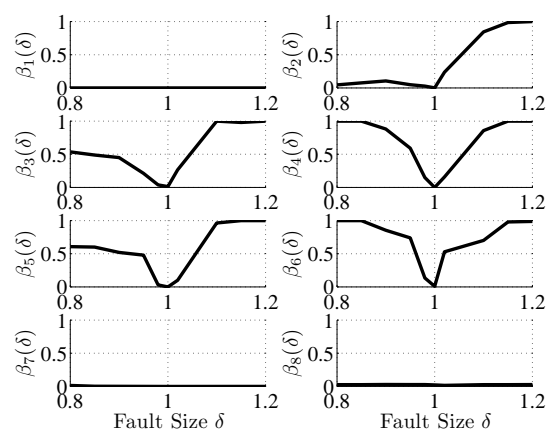

(a) Fault $\Delta_{y_{p_{\text {ic }}}}$

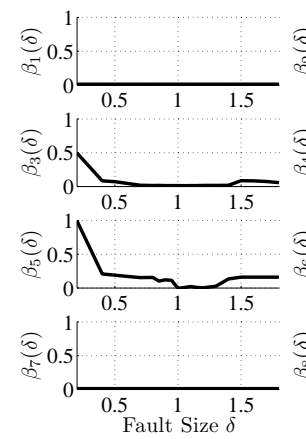

(b) Fault $\Delta_{u_{x_{\mathrm{th}}}}$

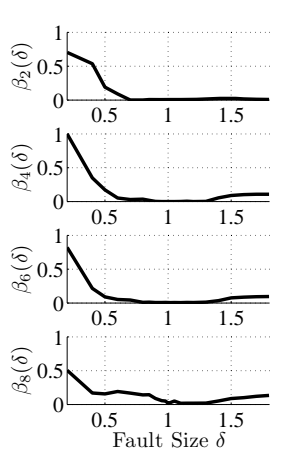

Figure 11: Power functions $\beta_{i}(\delta), i=1,2, \ldots, 8$, for faults $\Delta_{y_{p_{\text {ic }}}}$ and $\Delta_{u_{x_{\mathrm{th}}}}$.

power functions were estimated by means of the fraction of samples for which the corresponding test alarmed, i.e., where $d_{i}=1$.

As seen in both Figure 11 and Table 8, the powers of all tests are not ideal for all faults and all fault sizes. For example, some tests, e.g., $T_{2}$, respond only to sizes $\delta>1$ for some faults, and only to sizes $\delta<1$ for other faults. However, for instance fault $\Delta_{y_{p_{\text {ic }}}}$, results in a useful test power for almost all tests.

By considering the right most column in Table 8, it can be deduced that the average fault detection performances for all tests are comparable, but that tests $T_{4}, T_{5}, T_{6}$, and $T_{8}$, seem to be slightly better than the other tests. By considering the last row in Table 8, it can be deduced that the pressure sensor faults, $\Delta_{y_{p_{\mathrm{ic}}}}, \Delta_{y_{p_{\mathrm{im}}}}$, and $\Delta_{y_{p_{\mathrm{em}}}}$, seem to result in best overall averaged test 
power than all other faults. Faults $\Delta_{y_{T_{\mathrm{amb}}}}, \Delta_{u_{x_{\mathrm{th}}}}$, and $\Delta_{u_{x_{\mathrm{egr}}}}$, result in quite poor test power in comparison. This can also be seen in Figure 11.

The correspondence between the FSM in Table 4 and the averaged test powers in Table 8 when it comes to non-sensitive residual generators is good, in the sense that an empty entry in Table 4 always corresponds to a zero, or almost zero, entry in Table 8. However, the converse is not always true, since there are zero, or almost zero, entries in Table 8 where there is an " $\mathrm{x}$ " in Table 4. In particular, this holds for faults $\Delta_{y_{p_{\mathrm{amb}}}}$ and $\Delta_{u_{x_{\mathrm{vgt}}}}$. According to Table 4 , all residual generators may be sensitive to faults $\Delta_{y_{p_{\mathrm{amb}}}}$ and $\Delta_{u_{x_{\mathrm{vgt}}}}$. However, as indicated by Table 8, all tests do not respond to these faults.

\subsection{Performance of FDI-System}

The aim of this part of the evaluation is to investigate the detection and isolation performance of the complete FDI-system.

\subsubsection{Metrics}

To this end, the following metrics are considered.

Detection Time (DT): Time from fault injection to first detection by any test that may be sensitive to the fault.

Isolation Time (IT): Time from fault injection to first correct fault isolation statement.

Missed Detection Rate (MDR): The fraction of test runs for which the injected fault is not detected by any of the tests that may be sensitive to the fault.

Missed Isolation Rate (MIR): The fraction of test runs for which a correct fault isolation statement is not obtained.

False Detection Rate (FDR): The fraction of samples for which the injected fault is detected by a test that should not be sensitive to the fault, or a fault is detected by any test in a no-fault condition. 
Table 9: Specification of fault sizes used in the experimental evaluation.

\begin{tabular}{ll}
\hline Fault & Specification \\
\hline$\Delta_{y_{p_{\mathrm{amb}}}}$ & $y_{p_{\mathrm{amb}}}=0.5 \cdot p_{\mathrm{amb}}$ \\
$\Delta_{y_{T_{\mathrm{amb}}}}$ & $y_{T_{\mathrm{amb}}}=1.3 \cdot T_{\mathrm{amb}}$ \\
$\Delta_{y_{p_{\mathrm{ic}}}}$ & $y_{p_{\mathrm{ic}}}=1.2 \cdot p_{\text {ic }}$ \\
$\Delta_{y_{p_{\mathrm{im}}}}$ & $y_{p_{\mathrm{im}}}=0.9 \cdot p_{\mathrm{im}}$ \\
$\Delta_{y_{T_{\mathrm{im}}}}$ & $y_{T_{\mathrm{im}}}=0.7 \cdot T_{\mathrm{im}}$ \\
$\Delta_{y_{p_{\mathrm{em}}}}$ & $y_{p_{\mathrm{em}}}=0.8 \cdot p_{\mathrm{em}}$ \\
$\Delta_{u_{x_{\mathrm{th}}}}$ & $u_{x_{\mathrm{th}}}=0.3 \cdot u_{x_{\mathrm{th}}}$ \\
$\Delta_{u_{x_{\mathrm{egr}}}}$ & $u_{x_{\mathrm{egr}}}=0.4 \cdot u_{x_{\mathrm{egr}}}$ \\
$\Delta_{u_{x_{\mathrm{vgt}}}}$ & $u_{x_{\mathrm{vgt}}}=0.5 \cdot u_{x_{\mathrm{vgt}}}$
\end{tabular}

Note that all metrics are defined with respect to the complete FDI-system, and not in the context of the individual tests. This means, for instance, that a run in which only one out of several sensitive tests responds, will not be regarded as a missed detection. A situation where only one out of several possible tests responds falsely, will on the other hand be counted as a false detection. Also note that missed detections and missed isolations are counted on test run basis, whereas false detections are counted on sample basis.

It is also noted that with a correct fault isolation statement it is meant an isolation statement in accordance with the isolability matrix in Table 5. That is, when fault $\Delta_{y_{p_{\text {amb }}}}$ has occurred, the correct fault isolability statement is that either of the faults $\Delta_{y_{p_{\mathrm{amb}}}}$ or $\Delta_{u_{x_{\mathrm{vgt}}}}$ has occurred.

\subsubsection{Setup}

12 different data sets were used in this part of the evaluation in total. As in the previous study, the data sets contain measurements from drives with both high-way and city parts under different conditions. Each fault specified in Table 9 was injected abruptly after a fixed time one at a time in each of the 12 data sets. In essence there were in total 12 test runs per fault. The sizes of the faults, as specified in Table 9, were chosen in consultation with experienced engineers in order to be realistic for the diesel engine considered. 


\subsubsection{Results and Comments}

Table 10 gives the mean, minimum, and maximum, detection time (DT), mean, minimum, and maximum, isolation time (IT), as well as the missed detection rate (MDR), missed isolation rate (MIR), and false detection rate (FDR), for all considered faults. The detection times and isolation times are given in seconds.

First of all, it can be noted in Table 10 that all faults can be detected within reasonable time, meaning that there were no missed detections. However, ideal isolation statements were not obtained for all faults. Nevertheless, the injected fault was contained in each of the obtained isolation statement. The occurrence of missed isolations can be explained by the fact that the FSM in Table 4 used in the isolation scheme, see Section 5.3, does not completely reflect the fault sensitivity of the tests in the FDI-system. This was illustrated in Figures 9b and $10 \mathrm{~b}$ and will be considered in next section.

It is evident from Table 10 that the conclusion in Section 6.1.4 regarding the ability to detect the pressure sensor faults $\Delta_{y_{p_{\mathrm{ic}}}}, \Delta_{y_{p_{\mathrm{im}}}}$, and $\Delta_{y_{p_{\mathrm{em}}}}$ in a reliable way, is supported by Table 10. All of these faults result in comparatively short detection times, low rates of false detections, and can be isolated to a higher extent than the other faults. The same holds for the conclusions in Section 6.1.4 regarding the faults $\Delta_{y_{T_{\mathrm{amb}}}}$ and $\Delta_{u_{x_{\mathrm{egr}}}}$, which according to Table 10 results in longer detection times, and higher rates of false detection.

The absolute values of the metrics in Table 10 depend mainly on the value of the detection thresholds. The higher the detection thresholds, the lower the rate of false detection. The higher the rate of missed detection, and the longer the detection and isolation times, and vice versa. In addition, as said in Section 5.2, the detection and isolation times is affected by the size of the sliding windows used to collect samples for the residual evaluation.

\subsection{Final Tuning}

Until now, no specific tuning of the FDI-system has been performed. In this section it is illustrated how the FDI-system can be tuned in order to give lower 
Table 10: Summary of detection time (DT), isolation time (IT), missed detection rate (MDR), missed isolation rate (MIR), and false detection rate (FDR), for all considered faults. All time specifications are given in seconds.

\begin{tabular}{|c|c|c|c|c|c|c|c|c|c|c|}
\hline & & 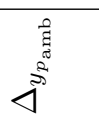 & 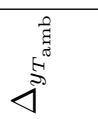 & $\underset{j}{\vec{s}}$ & $\underset{8}{\stackrel{5}{5}}$ & $\underset{8}{\stackrel{5}{5}}$ & 劳 & 营 & 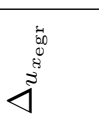 & 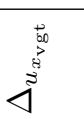 \\
\hline \multirow{3}{*}{ DT } & Mean & 49.1 & 78.4 & 33.2 & 41.1 & 86.5 & 39.2 & 66.5 & 75.0 & 90.9 \\
\hline & Min & 5.0 & 2.3 & 18.7 & 18.7 & 4.8 & 11.9 & 9.4 & 2.9 & 6.1 \\
\hline & Max & 83.6 & 35.9 & 72.5 & 115.0 & 290.5 & 61.3 & 166.8 & 116.9 & 144.3 \\
\hline \multirow{3}{*}{ IT } & Mean & - & - & 221.0 & 149.0 & - & 523.0 & 308.8 & - & - \\
\hline & Min & - & - & 97.0 & 96.6 & - & 261.4 & 227.2 & - & - \\
\hline & Max & - & - & 437.9 & 223.8 & - & 784.7 & 369.5 & - & - \\
\hline MDR & & 0 & 0 & 0 & 0 & 0 & 0 & 0 & 0 & 0 \\
\hline MIR & & 1 & 1 & 0.75 & 0.67 & 1 & 0.83 & 0.75 & 1 & 1 \\
\hline FDR & & 0.043 & 0.076 & 0.057 & 0.067 & 0.043 & 0.049 & 0.056 & 0.051 & 0.043 \\
\hline
\end{tabular}

rates of missed isolation for all faults.

As said in Section 6.2.3, the missed isolations is a direct consequence of the mismatch between the fault sensitivity as specified by the FSM used in the isolation process, and the actual fault sensitivity. There are at least two approaches for solving this issue. The first approach is to lower the detection thresholds. This would obviously resolve situations similar as those depicted in Figures 9b and 10b, where a test responds but the response is not sufficient in order for the test statistic to cross the threshold. However, this would also increase the amount of false detections. In addition, the situation where a test does not respond at all to a fault, is not handled.

The second approach is to adjust the FSM so that it represents the actual fault sensitivity of the tests. This can be done by exploiting the averaged test powers in Table 8 . The benefit with this approach is that the detection thresholds can be adjusted in order to achieve desired detection times, and desired rates of false and missed detections. The main drawback is that it may affect the overall detectability and isolability properties of the FDI-system, due to additional zeros in the adjusted FSM. See (Krysander, 2006, Chapter 11) for a more general treatment of this issue. Moreover, it should be noted that the adjustment of the FSM typically relies on estimated test power, which strongly 
Table 11: Fault signature matrix that is adjusted based on actual fault sensitivities.

\begin{tabular}{|c|c|c|c|c|c|c|c|c|c|}
\hline & ]$^{\text {है }}$ & ${ }^{\text {है }}$ & ji & ja & 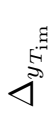 & वे & 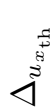 & $j^{50}$ & $j_{j}^{+\infty}$ \\
\hline$T_{1}$ & & $\mathrm{X}$ & & $\mathrm{X}$ & $\mathrm{X}$ & $\mathrm{X}$ & & $\mathrm{X}$ & $\mathrm{X}$ \\
\hline$T_{2}$ & $\mathrm{x}$ & $\mathrm{x}$ & $\mathrm{X}$ & $\mathrm{x}$ & & $\mathrm{x}$ & $\mathrm{x}$ & & $\mathrm{x}$ \\
\hline$T_{3}$ & $\mathrm{X}$ & $\mathrm{X}$ & $\mathrm{X}$ & $\mathrm{X}$ & $\mathrm{X}$ & $\mathrm{X}$ & $\mathrm{X}$ & & $\mathrm{X}$ \\
\hline$T_{4}$ & & & $\mathrm{X}$ & $\mathrm{X}$ & & & $\mathrm{X}$ & & \\
\hline$T_{5}$ & & & $\mathrm{X}$ & $\mathrm{X}$ & $\mathrm{X}$ & $\mathrm{X}$ & $\mathrm{X}$ & & \\
\hline$T_{6}$ & $\mathrm{x}$ & $\mathrm{X}$ & $\mathrm{X}$ & & $\mathrm{X}$ & $\mathrm{x}$ & $\mathrm{x}$ & $\mathrm{x}$ & $\mathrm{x}$ \\
\hline$T_{7}$ & & $\mathrm{x}$ & & $\mathrm{x}$ & $\mathrm{x}$ & $\mathrm{x}$ & & $\mathrm{x}$ & $\mathrm{x}$ \\
\hline$T_{8}$ & $\mathrm{x}$ & $\mathrm{x}$ & & $\mathrm{X}$ & $\mathrm{X}$ & $\mathrm{X}$ & $\mathrm{X}$ & $\mathrm{X}$ & $\mathrm{x}$ \\
\hline
\end{tabular}

depends on the features of the available data.

\subsubsection{Results}

Both approaches were applied. However, the first approach did not give satisfactory results. Despite detection thresholds resulting in fault detection rates in the magnitude of 30-40\%, the resulting missed isolation rates were not lower for all faults.

Using the second approach, the averaged powers of the residual evaluation tests as given in Table 8 were used in order to adjust the entries of the FSM in Table 4. Specifically, each " $\mathrm{x}$ " in the FSM in Table 4 was removed if the corresponding entry in Table 8 was lower than 0.02 . The removed entries are marked with bold in Table 8. The adjusted FSM, for residual evaluators instead of the residual generators, is given in Table 11.

The resulting isolability matrix is shown in Table 12, which should be compared with the original isolability matrix given in Table 5 . It can be noted that the isolability in fact has increased in the sense that a larger fraction of the diagnosis requirement $\mathcal{F}$ in $(5)$ is fulfilled. Specifically, 58 of the 72 fault pairs in $\mathcal{F}$ can now be isolated from each other, in comparison with 56 before.

Results in accordance with Table 10 are given for the FDI-system with the adjusted FSM in Table 13. The same detection thresholds and data were used as in the evaluation presented in Table 10.

It can be seen in Table 13 that the missed isolation rate (MIR) is lower 
Table 12: Isolability matrix based on adjusted fault signature matrix.

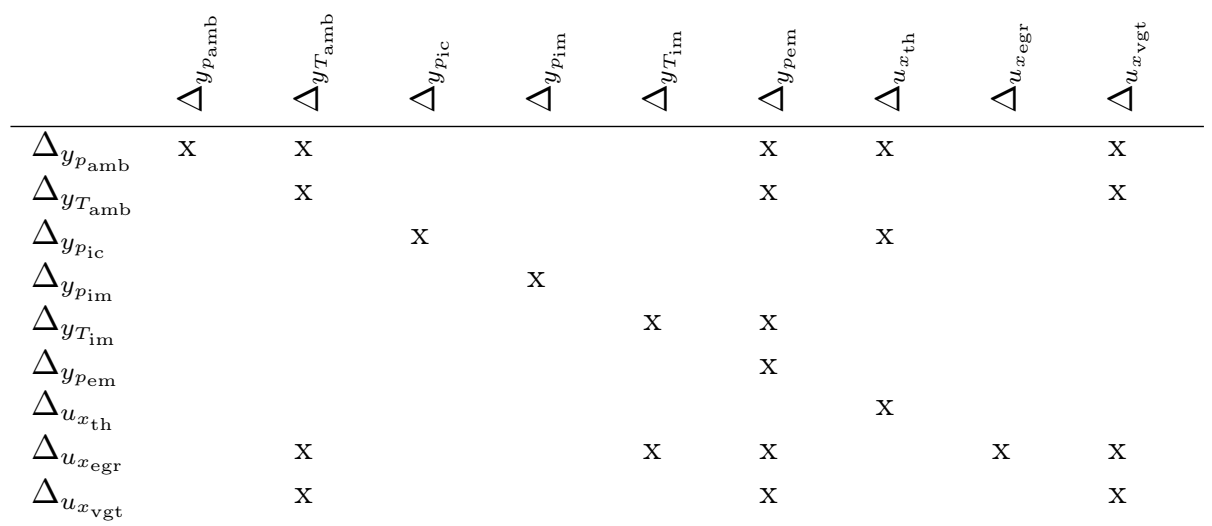

Table 13: Summary of detection time (DT), isolation time (IT), missed detection rate (MDR), missed isolation rate (MIR), and false detection rate (FDR), for all considered faults using the adjusted fault signature matrix. All time specifications are given in seconds.

\begin{tabular}{|c|c|c|c|c|c|c|c|c|c|c|}
\hline & & t" & 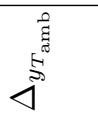 & $\underbrace{5}$ & $\frac{8}{s^{5}}$ & $\begin{array}{c}5 \\
\frac{5}{5}\end{array}$ & 离 & 常 & 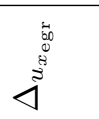 & 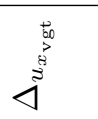 \\
\hline \multirow{3}{*}{ DT } & Mean & 48.1 & 82.9 & 33.2 & 41.1 & 87.0 & 39.2 & 66.5 & 77.8 & 90.7 \\
\hline & Min & 5.0 & 2.3 & 18.7 & 18.7 & 4.8 & 11.9 & 9.4 & 2.9 & 6.1 \\
\hline & Max & 83.6 & 35.9 & 72.5 & 115.0 & 290.5 & 61.3 & 166.8 & 116.9 & 144.3 \\
\hline \multirow{3}{*}{ IT } & Mean & 168.7 & 228.6 & 47.2 & 148.0 & 142.7 & 190.4 & 246.8 & 315.7 & 430.5 \\
\hline & Min & 45.5 & 173.3 & 28.5 & 96.6 & 142.7 & 57.1 & 62.0 & 5.3 & 129.8 \\
\hline & Max & 346.3 & 283.2 & 94.0 & 223.8 & 142.7 & 784.7 & 329.6 & 545.8 & 612.8 \\
\hline MDR & & 0 & 0 & 0 & 0 & 0 & 0 & 0 & 0 & 0 \\
\hline MIR & & 0.42 & 0.75 & 0 & 0.58 & 0.83 & 0.25 & 0.42 & 0.67 & 0.67 \\
\hline FDR & & 0.11 & 0.082 & 0.064 & 0.067 & 0.053 & 0.049 & 0.056 & 0.063 & 0.069 \\
\hline
\end{tabular}

for all faults, in comparison to Table 10. In addition, the isolation times are lower for all faults, and for some faults, e.g., $\Delta_{y_{p_{\mathrm{ic}}}}$, the difference is significant. Furthermore, the detection times are identical, or comparable, with those given in Table 10. It may be noted that there is a slight increase in false detection rate. This is a direct consequence of the additional empty entries in the adjusted FSM shown in Table 11 since each detection of a fault by a test whose corresponding entry in Table 11 has been removed now counts as a false detection. 


\section{Conclusions}

It has been illustrated how an FDI-system for an automotive diesel engine can be designed by application of a generic automated design methodology. No specific adaptation of the methodology to the automotive diesel engine system was made. Through the application, it has been empirically shown that employment of mixed causality substantially increased the number of realizable residual generators. Foremost, this leads to increased fault isolability as is evident by comparison of Tables 5 and 7. Moreover, it has been demonstrated how model errors of time-varying nature and magnitude can be handled in the framework of statistical likelihood-based residual evaluation. Illustrations are given in Figures 9 and 10.

The FDI-system, and thus the potential of the automated design methodology, has been evaluated using road and test-bed measurements. The fault sensitivities of the individual fault detection tests have been investigated by means of the estimated averaged test power (8). It was concluded that the fault sensitivity indicated in the FSM in Table 4 does not fully correspond to the fault sensitivity as given by the averaged test powers shown in Table 8. Specifically, this results in high missed isolation rates. It has been illustrated that an adjustment of the original FSM by utilization of the averaged test powers, yielding the adjusted FSM in Table 11, results in an FDI-system that is capable of isolating more faults from each other, as can be seen by the comparison of Tables 12 and 5. In addition, this also resulted in increased fault isolation performance, in terms of substantially lower missed isolation rates and shorter isolation times, in comparison with the original FSM, which can be seen by the comparison of Tables 13 and 10.

\section{Acknowledgment}

This work was sponsored by Scania and VINNOVA (Swedish Governmental Agency for Innovation Systems). The work by Erik Frisk and Mattias Krysander 
has been partially supported by the Swedish Research Council within The Linnaeus Center CADICS.

\section{Appendix A. Diesel Engine Model}

The section describes the diesel engine model. The model equations are stated below. The variables, parameters, functions, sensors and actuators, and faults, are described in Tables A.14, A.15, A.16, A.17, and A.18, respectively. A detailed description of the model can be found in Wahlström and Eriksson (2011), and a Matlab/Simulink implementation may be downloaded from Wahlström and Eriksson (2012).

$$
\begin{aligned}
e_{1}: & \dot{p}_{\mathrm{ic}}=\frac{R_{\mathrm{a}} T_{\mathrm{im}}}{V_{\mathrm{ic}}}\left(W_{\mathrm{c}}-W_{\mathrm{th}}\right) \\
e_{2}: & \dot{p}_{\mathrm{im}}=\frac{R_{\mathrm{a}} T_{\mathrm{im}}}{V_{\mathrm{im}}}\left(W_{\mathrm{th}}+W_{\mathrm{egr}}-W_{\mathrm{ei}}\right) \\
e_{3}: & \dot{p}_{\mathrm{em}}=\frac{R_{\mathrm{e}} T_{\mathrm{em}}}{V_{\mathrm{em}}}\left(W_{\mathrm{eo}}-W_{\mathrm{egr}}-W_{\mathrm{t}}\right) \\
& +\frac{R_{\mathrm{e}}}{V_{\mathrm{em}} c_{\mathrm{ve}}}\left(W_{\mathrm{in}} c_{\mathrm{ve}}\left(T_{\mathrm{em}, \mathrm{in}}-T_{\mathrm{em}}\right)+R_{\mathrm{e}}\left(T_{\mathrm{em}, \mathrm{in}} W_{\mathrm{in}}-T_{\mathrm{em}} W_{\mathrm{out}}\right)\right) \\
e_{4}: & \dot{T}_{\mathrm{em}}=\frac{R_{\mathrm{e}} T_{\mathrm{em}}}{p_{\mathrm{em}} V_{\mathrm{em}} c_{\mathrm{ve}}}\left(W_{\mathrm{in}} c_{\mathrm{ve}}\left(T_{\mathrm{em}, \mathrm{in}}-T_{\mathrm{em}}\right)\right. \\
& \left.+R_{\mathrm{e}}\left(T_{\mathrm{em}, \mathrm{in}} W_{\mathrm{in}}-T_{\mathrm{em}} W_{\mathrm{out}}\right)\right) \\
e_{5}: & W_{\mathrm{in}}=\max \left(W_{\mathrm{eo}}, 0\right)+\max \left(-W_{\mathrm{egr}}, 0\right)+\max \left(-W_{\mathrm{t}}, 0\right) \\
e_{6}: & W_{\mathrm{out}}=\max \left(-W_{\mathrm{eo}}, 0\right)+\max \left(W_{\mathrm{egr}}, 0\right)+\max \left(W_{\mathrm{t}}, 0\right) \\
e_{7}: & W_{\mathrm{th}}=\frac{p_{\mathrm{ic}} A_{\mathrm{th}, \mathrm{max}}}{\sqrt{T_{\mathrm{im}} R_{\mathrm{a}}}} \Psi_{\mathrm{th}}^{\gamma_{\mathrm{th}}}\left(\Pi_{\mathrm{th}}\right) f_{\mathrm{th}}\left(x_{\mathrm{th}}\right) \\
e_{8}: & \Pi_{\mathrm{th}}=f_{\Pi_{\mathrm{th}}}\left(p_{\mathrm{im}}, p_{\mathrm{ic}}\right) \\
e_{9}: & W_{\mathrm{ei}}=\frac{\eta_{\mathrm{vol}} p_{\mathrm{im}} n_{\mathrm{e}} V_{d}}{120 R_{\mathrm{a}} T_{\mathrm{im}}} \\
e_{10}: & \eta_{\mathrm{vol}}=c_{\mathrm{vol} 1} \frac{r_{\mathrm{c}}-\left(\frac{p_{\mathrm{em}}}{p_{\mathrm{im}}}\right)}{r_{\mathrm{c}}-1}+c_{\mathrm{vol} 2} W_{\mathrm{f}}^{2}+c_{\mathrm{vol} 3} W_{\mathrm{f}}+c_{\mathrm{vol} 4} \\
e_{11}: & W_{\mathrm{f}}=\frac{10^{-6}}{120} \delta n_{\mathrm{e}} n_{\mathrm{cyl}} \\
e_{12}: & W_{\mathrm{eo}}=W_{\mathrm{f}}+W_{\mathrm{ei}}
\end{aligned}
$$




$$
\begin{aligned}
& e_{13}: \quad T_{\mathrm{e}}=T_{\mathrm{im}}+\frac{q_{H V} f_{T_{\mathrm{e}} W_{\mathrm{f}}}\left(W_{\mathrm{f}}\right) f_{T_{\mathrm{e}} n_{\mathrm{e}}}\left(n_{\mathrm{e}}\right)}{c_{\mathrm{pe}} W_{\mathrm{eo}}} \\
& e_{14}: \quad T_{\mathrm{em}, \mathrm{in}}=T_{\mathrm{amb}}+\left(T_{\mathrm{e}}-T_{\mathrm{amb}}\right) \exp \left(-\frac{h_{\text {tot }} \pi d_{\text {pipe }} l_{\text {pipe }} n_{\text {pipe }}}{W_{\mathrm{eo}} c_{\mathrm{pe}}}\right) \\
& e_{15}: \quad W_{\mathrm{egr}}=f_{W_{\mathrm{egr}}}\left(p_{\mathrm{im}}, p_{\mathrm{em}}, T_{\mathrm{em}}, x_{\mathrm{egr}}\right) \\
& e_{16}: \quad \dot{\omega}_{\mathrm{t}}=\frac{P_{\mathrm{t}} \eta_{m}-P_{\mathrm{c}}}{J_{\mathrm{t}} \omega_{\mathrm{t}}} \\
& e_{17}: \quad P_{\mathrm{t}} \eta_{\mathrm{m}}=\eta_{\mathrm{tm}} W_{\mathrm{t}} c_{\mathrm{pe}} T_{\mathrm{em}}\left(1-\Pi_{\mathrm{t}}^{1-1 / \gamma_{\mathrm{e}}}\right) \\
& e_{18}: \quad \eta_{\mathrm{tm}}=\eta_{\mathrm{tm}, \mathrm{BSR}}(B S R) \eta_{\mathrm{tm}, \omega_{\mathrm{t}}}\left(\omega_{\mathrm{t}}\right) \eta_{\mathrm{tm}, x_{\mathrm{vgt}}}\left(x_{\mathrm{vgt}}\right) \\
& e_{19}: \quad B S R=\frac{R_{\mathrm{t}} \omega_{\mathrm{t}}}{\sqrt{2 c_{\mathrm{pe}} T_{\mathrm{em}}\left(1-\Pi_{\mathrm{t}}^{1-1 / \gamma_{\mathrm{e}}}\right)}} \\
& e_{20}: \quad \Pi_{\mathrm{t}}=\frac{p_{\mathrm{t}}}{p_{\mathrm{em}}} \\
& e_{21}: \quad W_{\mathrm{t}}=\frac{A_{\mathrm{vgt}, \max } p_{\mathrm{em}}}{\sqrt{T_{\mathrm{em}} R_{\mathrm{e}}}} f_{\Pi_{\mathrm{t}}}\left(\Pi_{\mathrm{t}}\right) f_{\omega_{\mathrm{t}}}\left(\omega_{\mathrm{t}, \mathrm{corr}}\right) f_{\mathrm{vgt}}\left(x_{\mathrm{vgt}}\right) \\
& e_{22}: \quad \omega_{t, c o r r}=\frac{\omega_{\mathrm{t}}}{100 \sqrt{T_{\mathrm{em}}}} \\
& e_{23}: \quad P_{\mathrm{c}}=\frac{W_{\mathrm{c}} c_{p a} T_{\mathrm{bc}}}{\eta_{\mathrm{c}}}\left(\Pi_{\mathrm{c}}^{1-1 / \gamma_{\mathrm{a}}}-1\right) \\
& e_{24}: \quad \Pi_{\mathrm{c}}=\frac{p_{\mathrm{ic}}}{p_{\mathrm{bc}}} \\
& e_{25}: \quad \eta_{\mathrm{c}}=\eta_{c, W}\left(W_{c, \text { corr }}, \Pi_{\mathrm{c}}\right) \eta_{c, \Pi}\left(\Pi_{\mathrm{c}}\right) \\
& e_{26}: \quad W_{\mathrm{c}, \mathrm{corr}}=\frac{\sqrt{\left(T_{\mathrm{bc}} / T_{\mathrm{ref}}\right)}}{\sqrt{\left(p_{\mathrm{bc}} / p_{\mathrm{ref}}\right)}} W_{\mathrm{c}} \\
& e_{27}: \quad W_{\mathrm{c}}=\frac{p_{\mathrm{bc}} \pi R_{\mathrm{c}}^{3} \omega_{\mathrm{t}}}{R_{\mathrm{a}} T_{\mathrm{bc}}} \Phi_{\mathrm{c}} \\
& e_{28}: \quad \Phi_{\mathrm{c}}=\frac{k_{c 1}-k_{c 3} \Psi_{\mathrm{c}}}{k_{c 2}-\Psi_{\mathrm{c}}} \\
& e_{29}: \quad k_{c 1}=k_{c 11}\left(\min \left(M a, M a_{\max }\right)\right)^{2}+k_{c 12} \min \left(M a, M a_{\max }\right)+k_{c 13} \\
& e_{30}: k_{c 2}=k_{c 21}\left(\min \left(M a, M a_{\max }\right)\right)^{2}+k_{c 22} \min \left(M a, M a_{\max }\right)+k_{c 23} \\
& e_{31}: \quad k_{c 3}=k_{c 31}\left(\min \left(M a, M a_{\max }\right)\right)^{2}+k_{c 32} \min \left(M a, M a_{\max }\right)+k_{c 33} \\
& e_{32}: \quad M a=\frac{R_{\mathrm{c}} \omega_{\mathrm{t}}}{\sqrt{\gamma_{\mathrm{a}} R_{\mathrm{a}} T_{\mathrm{bc}}}} \\
& e_{33}: \quad \Psi_{\mathrm{c}}=\frac{2 c_{p a} T_{\mathrm{bc}}\left(\Pi_{\mathrm{c}}^{1-1 / \gamma_{\mathrm{a}}}-1\right)}{R_{\mathrm{c}}^{2} \omega_{\mathrm{t}}^{2}}
\end{aligned}
$$




$$
\begin{array}{ll}
e_{34}: & p_{\mathrm{bc}}=p_{\mathrm{amb}} \\
e_{35}: & T_{\mathrm{bc}}=T_{\mathrm{amb}} \\
e_{36}: & y_{p_{\mathrm{amb}}}=p_{\mathrm{amb}}+\Delta_{y_{p_{\mathrm{amb}}}} \\
e_{37}: & y_{T_{\mathrm{amb}}}=T_{\mathrm{amb}}+\Delta_{y_{T_{\mathrm{amb}}}} \\
e_{38}: & y_{p_{\mathrm{ic}}}=p_{\mathrm{ic}}+\Delta_{y_{p_{\mathrm{ic}}}} \\
e_{39}: & y_{p_{\mathrm{im}}}=p_{\mathrm{im}}+\Delta_{y_{p_{\mathrm{im}}}} \\
e_{40}: & y_{T_{\mathrm{im}}}=T_{\mathrm{im}}+\Delta_{y_{T_{\mathrm{im}}}} \\
e_{41}: & y_{p_{\mathrm{em}}}=p_{\mathrm{em}}+\Delta_{y_{p_{\mathrm{em}}}} \\
e_{42}: & u_{x_{\mathrm{th}}}=x_{\mathrm{th}}+\Delta_{u_{x_{\mathrm{th}}}} \\
e_{43}: & u_{x_{\mathrm{egr}}}=x_{\mathrm{egr}}+\Delta_{u_{x_{\mathrm{egr}}}} \\
e_{44}: & u_{x_{\mathrm{vgt}}}=x_{\mathrm{vgt}}+\Delta_{u_{x_{\mathrm{vgt}}}} \\
e_{45}: & u_{\delta}=\delta \\
e_{46}: & y_{n_{\mathrm{e}}}=n_{\mathrm{e}}
\end{array}
$$

\section{References}

I. M. Al-Salami, S. X. Ding, and P. Zhang. Statistical based residual evaluation for fault detection in networked control systems. In Proceedings of Workshop on Advances Control and Diagnosis, Nancy, France, 2006. Nancy University.

M. Basseville and I. V. Nikiforov. Detection of Abrupt Changes - Theory and Application. Prentice-Hall, 1993.

M. Blanke, M. Kinnaert, J. Lunze, and M. Staroswiecki. Diagnosis and FaultTolerant Control. Springer, second edition, 2006.

M. R. Blas and M. Blanke. Stereo vision with texture learning for fault-tolerant automatic baling. Computers and Electronics in Agriculture, 75(1):159 - 68, 2011. 
Table A.14: Variables in the Model.

\begin{tabular}{|c|c|c|}
\hline Variable & Description & Unit \\
\hline$p_{\text {ic }}$ & Intercooler pressure & $\mathrm{Pa}$ \\
\hline$p_{\text {im }}$ & Intake manifold pressure & $\mathrm{Pa}$ \\
\hline$p_{\mathrm{em}}$ & Exhaust manifold pressure & $\mathrm{Pa}$ \\
\hline$p_{\mathrm{bc}}$ & Pressure before compressor & $\mathrm{Pa}$ \\
\hline$p_{\mathrm{amb}}$ & Ambient pressure & $\mathrm{Pa}$ \\
\hline$T_{\mathrm{em}}$ & Exhaust manifold temperature & $\mathrm{K}$ \\
\hline$T_{\mathrm{em}, \mathrm{in}}$ & Exhaust manifold in temperature & $\mathrm{K}$ \\
\hline$T_{\mathrm{im}}$ & Intake manifold temperature & $\mathrm{K}$ \\
\hline$T_{\mathrm{amb}}$ & Ambient temperature & $\mathrm{K}$ \\
\hline$T_{\mathrm{e}}$ & Cylinder temperature & $\mathrm{K}$ \\
\hline$T_{\mathrm{bc}}$ & Temperature before compressor & $\mathrm{K}$ \\
\hline$W_{\mathrm{c}}$ & Mass-flow through compressor & $\mathrm{kg} / \mathrm{s}$ \\
\hline$W_{\mathrm{th}}$ & Mass-flow through intake throttle & $\mathrm{kg} / \mathrm{s}$ \\
\hline$W_{\text {egr }}$ & EGR mass-flow & $\mathrm{kg} / \mathrm{s}$ \\
\hline$W_{\mathrm{ei}}$ & Mass-flow into engine & $\mathrm{kg} / \mathrm{s}$ \\
\hline$W_{\text {eo }}$ & Mass-flow out from engine & $\mathrm{kg} / \mathrm{s}$ \\
\hline$W_{\mathrm{t}}$ & Mass-flow through turbine & $\mathrm{kg} / \mathrm{s}$ \\
\hline$W_{\text {in }}$ & Mass-flow into exhaust manifold & $\mathrm{kg} / \mathrm{s}$ \\
\hline$W_{\text {out }}$ & Mass-flow out from exhaust manifold & $\mathrm{kg} / \mathrm{s}$ \\
\hline$W_{\mathrm{f}}$ & Fuel mass-flow & $\mathrm{kg} / \mathrm{s}$ \\
\hline$W_{\mathrm{c}, \mathrm{corr}}$ & Corrected mass-flow through compressor & $\mathrm{kg} / \mathrm{s}$ \\
\hline$x_{\mathrm{th}}$ & Throttle position & $\%$ \\
\hline$x_{\text {egr }}$ & EGR-valve position & $\%$ \\
\hline$x_{\mathrm{vgt}}$ & VGT-valve position & $\%$ \\
\hline$\Pi_{\text {th }}$ & Pressure ratio throttle & - \\
\hline$\Pi_{\mathrm{t}}$ & Pressure ratio turbine & - \\
\hline$\Pi_{\mathrm{c}}$ & Pressure ratio compressor & - \\
\hline$\eta_{\mathrm{vol}}$ & Volumetric efficiency & $\%$ \\
\hline$\eta_{\mathrm{tm}}$ & Fictive turbine efficiency & $\%$ \\
\hline$\eta_{\mathrm{c}}$ & Compressor efficiency & $\%$ \\
\hline$n_{\mathrm{e}}$ & Engine speed & $\mathrm{rpm}$ \\
\hline$\delta$ & Mass of injected fuel & $\mathrm{mg} /$ cycle \\
\hline$\omega_{\mathrm{t}}$ & Turbine speed & $\mathrm{rpm}$ \\
\hline$\omega_{\mathrm{t}, \mathrm{corr}}$ & Corrected turbine speed & $\mathrm{rpm}$ \\
\hline$P_{\mathrm{t} \eta_{m}}$ & Turbine power correction factor & - \\
\hline$P_{\mathrm{c}}$ & Compressor power & $\mathrm{W}$ \\
\hline$B S R$ & Blade tip ratio & - \\
\hline$\Psi_{\mathrm{c}}$ & Volumetric flow coefficient & $\%$ \\
\hline$M a$ & Mac number ring orifice of compressor & $\%$ \\
\hline$\Phi_{\mathrm{c}}$ & Energy transfer coefficient - & \\
\hline$k_{\mathrm{c} 1}$ & Compressor flow coefficient & - \\
\hline$k_{\mathrm{c} 2}$ & Compressor flow coefficient & - \\
\hline$k_{\mathrm{c} 3}$ & Compressor flow coefficient & - \\
\hline
\end{tabular}


Table A.15: Parameters in the Model.

\begin{tabular}{|c|c|}
\hline Parameter & Description \\
\hline$R_{\mathrm{a}}$ & Ideal gas constant for air \\
\hline$R_{\mathrm{e}}$ & Ideal gas constant for exhaust air \\
\hline$R_{\mathrm{t}}$ & Ideal gas constant for gas in turbine \\
\hline$R_{\mathrm{c}}$ & Ideal gas constant for gas in compressor \\
\hline$V_{\text {ic }}$ & Intercooler manifold volume \\
\hline$V_{\mathrm{im}}$ & Intake manifold volume \\
\hline$V_{\mathrm{em}}$ & Exhaust manifold volume \\
\hline$V_{\mathrm{d}}$ & Engine displacement volume \\
\hline$A_{\mathrm{th}, \max }$ & Maximum throttle area \\
\hline$A_{\mathrm{vgt}, \max }$ & Maximum VGT-valve area \\
\hline$\gamma_{\text {th }}$ & Specific heat capacity ratio for gas in throttle \\
\hline$\gamma_{\mathrm{e}}$ & Specific heat capacity ratio for exhaust gas \\
\hline$\gamma_{\mathrm{a}}$ & Specific heat capacity ratio for air \\
\hline$r_{\mathrm{c}}$ & Compression ratio \\
\hline$c_{\mathrm{ve}}$ & Specific heat capacity at constant volume in exhaust gas \\
\hline$c_{\mathrm{pe}}$ & Specific heat capacity at constant pressure in exhaust gas \\
\hline$c_{\mathrm{pa}}$ & Specific heat capacity at constant pressure in air \\
\hline$c_{\mathrm{vol} 1}, c_{\mathrm{vol} 2}, c_{\mathrm{vol} 3}, c_{\mathrm{vol} 4}$ & Volumetric efficiency constants \\
\hline$n_{\mathrm{cyl}}$ & Number of cylinders \\
\hline$q_{\mathrm{HV}}$ & Heating value of fuel \\
\hline$h_{\text {tot }}$ & Total heat transfer constant \\
\hline$d_{\text {pipe }}$ & Exhaust pipe diameter \\
\hline$l_{\text {pipe }}$ & Exhaust pipe length \\
\hline$n_{\text {pipe }}$ & Number of exhaust pipes \\
\hline$J_{\mathrm{t}}$ & Turbo inertia \\
\hline$B S R_{\mathrm{opt}}$ & Optimum blade tip ratio \\
\hline$p_{\mathrm{t}}$ & Turbine pressure \\
\hline$T_{\text {ref }}$ & Compressor air temperature reference \\
\hline$p_{\text {ref }}$ & Compressor air pressure reference \\
\hline$M a_{\max }$ & Maximum mac number ring orifice of compressor \\
\hline$k_{\mathrm{c} 11}, k_{\mathrm{c} 12}, k_{\mathrm{c} 13}$ & Compressor flow constants \\
\hline$k_{\mathrm{c} 21}, k_{\mathrm{c} 22}, k_{\mathrm{c} 23}$ & Compressor flow constants \\
\hline$k_{\mathrm{c} 31}, k_{\mathrm{c} 32}, k_{\mathrm{c} 33}$ & Compressor flow constants \\
\hline
\end{tabular}


Table A.16: Functions used in the Model.

\begin{tabular}{ll}
\hline Function & Description \\
\hline $\max ()$ & Maximum value \\
$\min ()$ & Minimum value \\
$\exp ()$ & Exponential function \\
$\Psi_{\mathrm{th}}()$ & Throttle flow Psi-function \\
$f_{\mathrm{th}}()$ & Throttle actuator function \\
$f_{\Pi_{\mathrm{th}}}()$ & Throttle pressure ratio function \\
$f_{T_{\mathrm{e}} W_{\mathrm{f}}}()$ & Cylinder temperature factor from mass-flow \\
$f_{T_{\mathrm{e} n_{\mathrm{e}}}}()$ & Cylinder temperature factor from engine speed \\
$f_{W_{\mathrm{egr}}}()$ & EGR-flow function \\
$\eta_{\mathrm{tm}, \mathrm{BSR}}()$ & Turbine efficiency factor from blade tip ratio \\
$\eta_{\mathrm{tm}, \omega_{\mathrm{t}}}()$ & Turbine efficiency factor from turbine speed \\
$\eta_{\mathrm{tm}, x_{\mathrm{vgt}}}()$ & Turbine efficiency factor from VGT-position \\
$f_{\Pi_{\mathrm{t}}}()$ & Turbine mass-flow factor from pressure ratio \\
$f_{\omega_{\mathrm{t}}}()$ & Turbine mass-flow factor from turbine speed \\
$f_{\mathrm{vgt}}()$ & Turbine mass-flow factor from VGT-position \\
$\eta_{\mathrm{c}, \mathrm{W}}()$ & Compressor efficiency factor from mass-flow \\
$\eta_{\mathrm{c}, \Pi}()$ & Compressor efficiency factor from pressure ratio \\
\hline
\end{tabular}

Table A.17: Sensors and Actuators in the Model.

\begin{tabular}{ll}
\hline Sensor & Description \\
\hline$y_{n_{\mathrm{e}}}$ & Engine speed sensor \\
$y_{p_{\mathrm{amb}}}$ & Ambient temperature sensor \\
$y_{T_{\mathrm{amb}}}$ & Ambient pressure sensor \\
$y_{p_{\mathrm{ic}}}$ & Inter-cooler pressure sensor \\
$y_{p_{\mathrm{im}}}$ & Inlet manifold pressure sensor \\
$y_{T_{\mathrm{im}}}$ & Inlet manifold temperature sensor \\
$y_{p_{\mathrm{em}}}$ & Exhaust manifold pressure sensor \\
\hline Actuator & Description \\
\hline$u_{x_{\mathrm{th}}}$ & Throttle position actuator \\
$u_{x_{\mathrm{egr}}}$ & EGR-valve position actuator \\
$u_{x_{\mathrm{vgt}}}$ & VGT-valve position actuator \\
$u_{\rho}$ & Injected fuel actuator \\
\hline
\end{tabular}


Table A.18: Faults in the Model.

\begin{tabular}{ll}
\hline Fault & Description \\
\hline$\Delta_{y_{p_{\mathrm{amb}}}}$ & Ambient pressure sensor fault \\
$\Delta_{y_{T_{\mathrm{amb}}}}$ & Ambient temperature sensor fault \\
$\Delta_{y_{p_{\mathrm{ic}}}}$ & Intercooler pressure sensor fault \\
$\Delta_{y_{p_{\mathrm{im}}}}$ & Intake manifold pressure sensor fault \\
$\Delta_{y_{T_{\mathrm{im}}}}$ & Intake manifold temperature sensor fault \\
$\Delta_{y_{p_{\mathrm{em}}}}$ & Exhaust manifold pressure sensor fault \\
$\Delta_{u_{x_{\mathrm{th}}}}$ & Throttle position actuator fault \\
$\Delta_{u_{x_{\mathrm{egr}}}}$ & EGR-valve position actuator fault \\
$\Delta_{u_{x_{\mathrm{vgt}}}}$ & VGT-valve position actuator fault \\
\hline
\end{tabular}

California EPA. Sections 1971.1, 1968.2, and 1971.5 of title 13, california code of regulations: HD OBD and OBD II regulations. http://www.arb.ca.gov/msprog/obdprog/hdobdreg.htm, 2010. California Environmental Protection Agency, Air Resources Board.

F. Caponetti, M. R. Blas, and M. Blanke. Stochastic automata for outdoor semantic mapping using optimised signal quantisation. Control Engineering Practice, 19(3):223-233, 2011.

G. Casella and R. L. Berger. Statistical Inference. Duxbury Press, second edition, 2001.

J. P. Cassar and M. Staroswiecki. A structural approach for the design of failure detection and identification systems. In Proceedings of IFAC Control Ind. Syst., pages 841-846, Belfort, France, 1997.

V. Cocquempot, R. Izadi-Zamanabadi, M. Staroswiecki, and M. Blanke. Residual generation for the ship benchmark using structural approach. In Proceedings of the UKACC International Conference on Control '98, pages 14801485, September 1998.

J. de Kleer and B. C Williams. Diagnosing multiple faults. Artificial Intelligence, 32(1):97-130, 1987.

D. Dustegor, V. Cocquempot, and M. Staroswiecki. Structural analysis for 
residual generation: Towards implementation. In Proceedings of the 2004 IEEE Inter. Conf. on Control App., pages 1217-1222, 2004.

European Parliament. Regulation No 595/2009 of the european parliament and of the council of 18 june 2009 on type-approval of motor vehicles and engines with respect to emissions from heavy duty vehicles (Euro VI) and on access to vehicle repair and maintenance information and amending Regulation (EC) No 715/2007 and Directive 2007/46/EC and repealing Directives 80/1269/EEC, 2005/55/EC and 2005/78/EC, 2009. European Parliament and the Council of the European Union.

P. Fogh Odgaard, J. Stoustrup, and M. Kinnaert. Fault tolerant control of wind turbines - a benchmark model. In Proceedings of the 7th IFAC Symposium on Fault Detection, Supervision and Safety of Technical Processes, pages 155160, Barcelona, Spain, 2009.

J. Gertler. Fault Detection and Diagnosis in Engineering Systems. Marcel Dekker, 1998.

R. Greiner, B. A. Smith, and R. W. Wilkerson. A correction to the algorithm in reiter's theory of diagnosis. Artificial Intelligence, 41:79-88, 1989.

E. Höckerdal, E. Frisk, and L. Eriksson. EKF-based adaptation of look-up tables with an air mass-flow sensor application. Control Engineering Practice, 19(5): 442-453, 2011a.

E. Höckerdal, E. Frisk, and L. Eriksson. Bias reduction in DAE estimators by model augmentation: Observability analysis and experimental evaluation. In 50th IEEE Conference on Decision and Control, Orlando, Florida, USA, $2011 b$.

R. Izadi-Zamanabadi. Structural analysis approach to fault fiagnosis with application to fixed-wing aircraft motion. In Proceedings of the 2002 American Control Conference, volume 5, pages 3949-3954, 2002. 
M. Krysander. Design and Analysis of Diagnosis Systems Using Structural Methods. PhD thesis, Linköpings universitet, June 2006.

M. Krysander and E. Frisk. Sensor placement for fault diagnosis. Systems, Man and Cybernetics, Part A: Systems and Humans, IEEE Transactions on, 38 (6):1398-1410, Nov. 2008.

M. Krysander, J. Åslund, and M. Nyberg. An efficient algorithm for finding minimal over-constrained sub-systems for model-based diagnosis. IEEE Trans. on Systems, Man, and Cybernetics - Part A: Systems and Humans, 38(1): 197-206, 2008.

S. Kullback and R. A. Leibler. On information and sufficiency. Annals of Mathematical Statistics, 22(1):79-86, 1951.

S. P. Lloyd. Least squares quantization in pcm. IEEE Transactions on Information Theory, 28(2):129-137, 1982.

J. B. MacQueen. Some methods for classification and analysis of multivariate observations. In Proceedings of 5th Berkeley Symposium on Mathematical Statistics and Probability, pages 281-297. University of California Press, 1967.

J. Mattingley and S. Boyd. Real-time convex optimization in signal processing. IEEE Signal Processing Magazine, 27(3):50-61, 2010.

J. Mattingley and S. Boyd. CVXGEN: a code generator for embedded convex optimization. Optimization and Engineering, 13(1):1-27, 2012.

J. Mohammadpour, M. Franchek, and K. Grigoriadis. A survey on diagnostic methods for automotive engines. International Journal of Engine Research, 13(1):41-64, 2012.

M. Nyberg. Automatic design of diagnosis systems with application to an automotive engine. Control Engineering Practice, 87(8):993-1005, 1999. 
M. Nyberg and M. Krysander. Statistical properties and design criterions for AI-based fault isolation. In Proceedings of the 17th IFAC World Congress, pages 7356-7362, Seoul, Korea, 2008.

M. Nyberg and T. Stutte. Model based diagnosis of the air path of an automotive diesel engine. Control Engineering Practice, 12(5):513 - 525, 2004.

Y. Peng, A. Youssouf, P. Arte, and M. Kinnaert. A complete procedure for residual generation and evaluation with application to a heat exchanger. IEEE Transactions on Control Systems Technology, 5(6):542 - 555, 1997.

S. Ploix, M. Desinde, and S. Touaf. Automatic design of detection tests in complex dynamic systems. In Proceedings of 16th IFAC World Congress, Prague, Czech Republic, 2005.

B. Pulido and C. Alonso-González. Possible conflicts: a compilation technique for consistency-based diagnosis. "IEEE Trans. on Systems, Man, and Cybernetics. Part B: Cybernetics", Special Issue on Diagnosis of Complex Systems, 34(5):2192-2206, 2004.

R. Reiter. A theory of diagnosis from first principles. Artificial Intelligence, 32: 57-95, 1987.

M. Staroswiecki. Fault Diagnosis and Fault Tolerant Control, chapter Structural Analysis for Fault Detection and Isolation and for Fault Tolerant Control. Encyclopedia of Life Support Systems, Eolss Publishers, Oxford, UK, 2002.

M. Staroswiecki and P. Declerck. Analytical redundancy in non-linear interconnected systems by means of structural analysis. In Proceedings of IFAC AIPAC'89, pages 51-55, Nancy, France, 1989.

C. Svärd. Methods for Automated Design of Fault Detection and Isolation Systems with Automotive Applications. $\mathrm{PhD}$ thesis, Linköping University, june 2012. Dissertation No. 1448. 
C. Svärd and M. Nyberg. Residual generators for fault diagnosis using computation sequences with mixed causality applied to automotive systems. IEEE Transactions on Systems, Man and Cybernetics, Part A: Systems and Humans, 40(6):1310-1328, 2010.

C. Svärd and M. Nyberg. Automated design of an FDI-system for the wind turbine benchmark. Journal of Control Science and Engineering, vol. 2012, 2012. Article ID 989873, 13 pages.

C. Svärd, M. Nyberg, and E. Frisk. A greedy approach for selection of residual generators. In Proceedings of the 22nd International Workshop on Principles of Diagnosis (DX-11), Murnau, Germany, 2011a.

C. Svärd, M. Nyberg, E. Frisk, and M. Krysander. Residual evaluation for fault diagnosis by data-driven analysis of non-stationary probability distributions. In Proceedings of the 50th IEEE Conference on Decision and Control and European Control Conference (CDC-ECC 2011), pages 95-102, 2011b.

L. Travé-Massuyès, T. Escobet, and X. Olive. Diagnosability analysis based on component-supported analytical redundancy. IEEE Trans. on Systems, Man, and Cybernetics - Part A: Systems and Humans, 36(6):1146-1160, November 2006.

United Nations. Regulation no. 49: Uniform provisions concerning the measures to be taken against the emission of gaseous and particulate pollutants from compressionignition engines for use in vehicles, and the emission of gaseous pollutants from positive-ignition engines fuelled with natural gas or liquefied petroleum gas for use in vehicles, 2008. ECE-R49.

United States EPA. 40 CFR Part 86, 89, et al: Control of air pollution from new motor vehicles and new motor vehicle engines; final rule. http://www.epa.gov/obd/regtech/heavy.htm, 2009. United States Environmental Protection Agency. 
O. Vainio, M. Renfors, and T. Saramaki. Recursive implementation of fir differentiators with optimum noise attenuation. Instrumentation and Measurement, IEEE Transactions on, 46(5):1202 -11207, oct 1997.

J. Wahlström and L. Eriksson. Modeling diesel engines with a variable-geometry turbocharger and exhaust gas recirculation by optimization of model parameters for capturing non-linear system dynamics. Proceedings of the Institution of Mechanical Engineers, Part D: Journal of Automobile Engineering, 225(7), July 2011.

J. Wahlström and L. Eriksson. Matlab/simulink model for a turbocharged diesel engine with EGR and VGT, 2012. http://www.fs.isy.liu.se/Software/TCDI_EGR_VGT/.

X. Wei, H. Liu, and Y. Qin. Fault diagnosis of rail vehicle suspension systems by using glrt. In Control and Decision Conference (CCDC), 2011 Chinese, pages $1932-1936,2011$.

A. Willsky and H. Jones. A generalized likelihood ratio approach to the detection and estimation of jumps in linear systems. Automatic Control, IEEE Transactions on, 21(1):108 - 112, 1976. 